\title{
Minimal Natural Supersymmetry after the LHC8
}

\author{
Manuel Dree** \\ BCTP and Physics Institute, University of Bonn, Bonn, Germany \\ Jong Soo $\mathrm{Kim}^{\dagger}$ \\ Instituto de Física Teórica UAM/CSIC, Madrid, Spain
}

\begin{abstract}
In this work, we present limits on natural supersymmetry scenarios based on searches in data taken during run 1 of the LHC. We consider a set of 22000 model points in a six dimensional parameter space. These scenarios are minimal in the sense of only keeping those superparticles relatively light that are required to cancel the leading quadratically divergent quantum corrections (from the top and QCD sector) to the Higgs mass in the Standard Model. The resulting mass spectra feature higgsinos as the lightest supersymmetric particle, as well as relatively light third generation $S U(2)$ doublet squarks and $S U(2)$ singlet stops and gluinos while assuming a Standard Model like Higgs boson. All remaining supersymmetric particles and Higgs bosons are assumed to be decoupled. We check each parameter set against a large number of LHC searches as implemented in the public code CheckMATE. These searches show a considerable degree of complementarity, i.e. in general many searches have to be considered in order to check whether a given scenario is allowed. We delineate allowed and excluded regions in parameter space. For example, we find that all scenarios where either $m_{\tilde{t}_{1}}<230 \mathrm{GeV}$ or $m_{\tilde{g}}<440 \mathrm{GeV}$ are clearly excluded, while all model points where $m_{\tilde{t}_{1}}>660 \mathrm{GeV}$ and $m_{\tilde{g}}>1180 \mathrm{GeV}$ remain allowed.
\end{abstract}

\section{INTRODUCTION}

Supersymmetry (SUSY) is one of the best motivated extensions of the standard model (SM); it stabilizes the gauge hierarchy against radiative corrections, and allows one-step unification of the gauge couplings of the SM [1. Assuming a discrete symmetry like $R$-parity [2], the lightest supersymmetric particle (LSP) is stable, and can make a good Dark Matter candidate 1]. This symmetry also implies that supersymmetric particles can only be produced in pairs and subsequently decay into SM particles and the LSP. Since the LSP escapes detection at both multipurpose detectors ATLAS and CMS, supersymmetric particle production can give rise to large missing transverse momentum, accompanied by high momentum jets and leptons.

The LHC detectors started to take data in 2010 at the center of mass energy of $7 \mathrm{TeV}$ and both collected about $5 \mathrm{fb}^{-1}$ of integrated luminosity. In 2012, the center of mass energy was increased to $\sqrt{s}=8 \mathrm{TeV}$ and at the end of the run, both ATLAS and CMS recorded about $20 \mathrm{fb}^{-1}$ of data. Unfortunately no significant excess above the SM expectation has been found, although a few 2 to $3 \sigma$ anomalies have been found, which can be explained in the framework of supersymmetry [3, 4. The null results of both experiments have been translated into strict bounds on extensions of the SM. These extensions include supersymmetric models with simplifying assumptions on the soft breaking sector such as in the mSUGRA/CMSSM model [5], as well as simplified models containing

\footnotetext{
* drees@th.physik.uni-bonn.de

$\dagger$ jong.kim@csic.es
}

only a few supersymmetric particles and couplings 6 . For example, assuming degenerate squark and gluino masses as well as a light neutralino LSP, squarks and gluinos below $1.8 \mathrm{TeV}$ are now excluded [7].

These search limits, together with the discovery of a relatively heavy SM-like Higgs boson [8, put some strain on a supersymmetric solution of the finetuning problem. As well known, in theories with exact supersymmetry the Higgs mass is completely unaffected by loop corrections. Once soft breaking terms are introduced, quadratically divergent corrections continue to cancel, unlike in the SM, but there are corrections to the squared Higgs mass parameters in the Lagrangian that scale with (combinations of) squares of these soft masses. At the same time corrections to the physical mass of the lightest $\mathrm{CP}$-even neutral Higgs boson, which at the tree-level is lighter than the $Z$ boson, only scale logarithmically with the soft SUSY breaking parameters, in particular the stop masses. A Higgs mass near $125 \mathrm{GeV}$ therefore requires relatively heavy stops, which in turn tends to require corrections to the Lagrangian parameters that are larger than the tree-level values.

Quantifying the resulting finetuning is far from trivial, however. Most analyses now define finetuning via the sensitivity measures first introduced in 9. Applying this to the weak scale Higgs mass parameters, which determine the size of the vacuum expectation values breaking the electroweak gauge symmetry, one finds that finetuning increases quadratically with the supersymmetric higgs(ino) mass parameter $\mu$ (at tree level), and with the soft supersymmetry breaking masses of the stop squarks (at one-loop level) and of the gluino (at two-loop level). Refs. [10 12] therefore define natural supersymmetry to contain rather light higgsinos, perhaps somewhat heavier stop squarks and still not very heavy gluinos. All other superparticles 
can be out of reach of the LHC without leading to undue finetuning, at least as defined in this manner.

We adopt this definition of natural supersymmetry in our analysis. It is based on an analysis of the Higgs potential at the electroweak scale, without assumptions about high-scale physics (e.g., boundary conditions for the soft terms); however, it makes the implicit assumption that weak-scale masses of the relevant superparticles are independent parameters. It is minimal in the sense of requiring the minimal number of relatively light particles needed to cancel the leading quadratically divergent radiative corrections to the mass of the Higgs boson in the SM. This feature allows a complete scan of the relevant parameter space using a cluster of a few thousand CPUs. This is why we focus on this "minimal natural supersymmetry" here 1

In particular, the first and second generation squarks are assumed to be decoupled. This avoids constraints from supersymmetric "flavor excitation" reactions like $q q^{\prime} \rightarrow \tilde{q} \tilde{q}^{\prime}$ as well as $q g \rightarrow \tilde{q} \tilde{g}$, whose cross sections can easily exceed those for $q \bar{q} \rightarrow \tilde{t} \tilde{t}^{*}, \tilde{g} \tilde{g}$ if $m_{\tilde{q}} \sim m_{\tilde{g}}, m_{\tilde{t}}$. At the same time, "natural SUSY" in this sense requires relatively light higgsinos, third generation squarks at the $\mathrm{TeV}$ scale or (preferably) below, and a gluino not far above $1 \mathrm{TeV}$. ATLAS and CMS have started to optimize searches for such scenarios and a relatively large number of "natural SUSY" searches have by now been published.

However, as already noted, for a given mass third generation scalars have much lower production cross sections than first generation squarks. In addition, the cascade decays of third generation scalars can be quite involved; in particular, top quarks in the final state decay into three jets, or into a single $b$-jet plus a charged lepton and a neutrino. These cascade decays tend to spread the signal over several final states. These two effects imply that limits on third generation sparticles are generally weaker than for first generation squarks. So far, experimental limits have been only derived on simplified natural SUSY scenarios involving at most three sparticles and with simplifying assumptions on the decay modes [15, 16]. In this paper we instead consider realistic natural SUSY scenarios which are consistent with low energy limits as well as the observation of the $125 \mathrm{GeV}$ Higgs boson.

\footnotetext{
1 The "most natural" spectrum does depend on how exactly finetuning is defined. For example, if the "fundamental" parameters are defined at a high scale where supersymmetry breaking is mediated to squarks and gluinos, the finetuning constraint on the gluino mass is often stronger than that on stop masses [13]. On the other hand, refs. 14] argue that stop masses well above $1 \mathrm{TeV}$ may well be natural once the total radiative corrections to the Higgs potential at the weak scale are considered, due to cancellations between different terms. Note that in these scenarios the weak-scale parameters are functions of - often fewer - "fundamental" parameters at the input scale, in which case the weak-scale parameters are not independent of each other.
}

Phenomenological studies of natural SUSY scenarios at the LHC have been performed in [17 22]. However, these earlier papers either did not investigate the whole parameter space of natural SUSY, or they did not use the entire available set of LHC searches, recast for a complete natural SUSY framework.

In this work, we look at the phenomenology of fairly general natural SUSY scenarios. We parameterize the spectrum of relevant superparticles with six free parameters: the masses of $S U(2)$ singlet ("righthanded") and doublet ("left-handed") stop squarks, the higgsino mass parameter, the gluino mass, the trilinear stop sector soft breaking parameter, and the ratio of vacuum expectation values of the two Higgs doublets. We define these parameters directly at the weak scale, without imposing any relations between them. We randomly sample this six dimensional parameter space with about 22,000 spectra, all of which have the correct Higgs mass and the lightest neutralino as the LSP, assuming flat priors. This set of model points covers a large number of collider signatures at the LHC. We simulate all signal processes and pass them to a fast detector simulation. These signal events are then confronted with current ATLAS and CMS searches. We consider the relevant searches for natural SUSY as well as inclusive SUSY searches at the LHC. We show allowed and excluded regions of parameter space. While we do not combine searches in a statistical sense, we show that many searches contribute to the final limits.

The remainder of this article is organized as follows. In Sect. II we define the natural SUSY scenario we consider, and qualitatively discuss its collider signatures. In Sect. III we first discuss the numerical tools employed for this study and then describe how we perform the scan. Our numerical results are shown in Sect. IV] We conclude in Sect. V].

\section{NATURAL SUPERSYMMETRY SETUP}

The goal of "natural" SUSY models is to minimize finetuning while accommodating a $125 \mathrm{GeV}$ Higgs boson as well as the negative results of searches for superparticles. The finetuning in question is associated with the spontaneous breaking of the electroweak gauge symmetry. In the Minimal Supersymmetric extension of the Standard Model (MSSM) the higgsino mass parameter $\mu$ is fixed by the minimization conditions of the Higgs potential [1],

$$
\mu^{2}=\frac{m_{H_{d}}^{2}-m_{H_{u}}^{2} \tan ^{2} \beta}{\tan ^{2} \beta-1}-\frac{1}{2} M_{Z}^{2} .
$$

Here $m_{H_{u, d}}^{2}$ are the squared soft supersymmetry breaking masses of the Higgs doublet giving masses to uptype and down-type quarks, respectively, $\tan \beta$ is the ratio of the vacuum expectation values of $H_{u}^{0}$ and $H_{d}^{0}$, and $M_{Z}$ is the mass of the $Z$ boson. In a "natural" 
theory each individual term on the right-hand side of eq.(1) should be at most of order $M_{Z}^{2}$. An immediate consequence is that $\mu$ should also be of order $M_{Z}$, leading to relatively light higgsinos in the spectrum.

The minimization condition (1) holds for running parameters defined at scale $Q_{\mathrm{EW}}$; a common choice is $Q_{\mathrm{EW}}=\sqrt{m_{\tilde{t}_{1}} m_{\tilde{t}_{2}}}$ where $\tilde{t}_{1}, \tilde{t}_{2}$ are the two stop mass eigenstates, since this approximately minimizes the leading radiative corrections to the Higgs potential 23. The soft breaking masses in eq.(1) are subject to radiative corrections. The leading one-loop corrections involve stop squarks and scale with $m_{\tilde{t}_{1,2}}^{2}$. Keeping these corrections small thus indicates that stop masses should be as small as possible [12. At two loop order, gluino loops also renormalize the soft breaking Higgs masses; keeping these corrections under control requires gluino masses not too much above the $\mathrm{TeV}$ scale [12].

It should be recognized that these arguments are somewhat qualitative. Obviously the upper bounds on higgsino, stop and gluino masses depend on how much finetuning one is willing to tolerate. Moreover, the precise definition of the "fundamental" parameters of the theory, including the energy scale at which they are defined, also matters [13. Here we follow the spirit of ref.12 and define our "minimal natural SUSY" scenario to have higgsinos with masses below $500 \mathrm{GeV}$, third generation scalar quarks with masses less than $1.5 \mathrm{TeV}$ and gluinos with mass below $3 \mathrm{TeV}$. Given the upper bound on the stop masses, the light $\mathrm{CP}-$ even Higgs boson $h$ can attain its observed mass near $125 \mathrm{GeV}$ only if the trilinear $\left|A_{t}\right|$ soft breaking term is quite large. The variable input parameters defining our natural SUSY scenario are listed in Table I.

In addition we assume a common large mass for the first and second generation squarks, $\tilde{b}_{R}$ squarks and all sleptons, which we fix to $m_{\tilde{f}}^{2}=1.5 \cdot 10^{7} \mathrm{GeV}^{2}$. This easily satisfies constraints from the null results of SUSY searches from ATLAS and CMS, avoids constraints from flavor changing neutral currents and alleviates bounds from $\mathrm{CP}$ violating processes [24]. Note that we only consider scenarios with $\tan \beta \leq 20$, so that $\tilde{b}$ loops are subdominant and $\tilde{b}_{R}$ can be made heavy. $\left(m_{\tilde{b}_{L}}=m_{\tilde{t}_{L}}\right.$ by gauge invariance.) We decouple the electroweak gauginos as well, setting $M_{1}=$ $M_{2}=3 \mathrm{TeV}$. Since the observed Higgs boson is SMlike, we are working in the decoupling limit with a large mass of the pseudoscalar Higgs, $m_{A}=2.5 \mathrm{TeV}$. The precise values of these masses basically do not matter for our analysis, as long as these particles are well beyond the range of LHC run-1. Since the bottom trilinear soft breaking coupling $A_{b}$ has only little impact on the phenomenology we set $A_{b}=0$ for simplicity. Since $m_{\tilde{b}_{R}}^{2} \gg m_{\tilde{b}_{L}}^{2}, L-R$ mixing in the sbottom sector is suppressed, in contrast to the stop sector, where the mass eigenstates generally are mixtures of $\tilde{t}_{L}$ and $\tilde{t}_{R}$ squarks. Finally, we assume $R$-parity to be conserved. This implies that the LSP, which is stable, must be electrically neutral; in the context of our scenario this means that the lightest neutralino must be the LSP.

In most cases making all sparticles heavy that are not involved in the simple finetuning argument outlined above should be conservative, in the sense that additional light superparticles lead to additional production channels which might exclude a scenario that is otherwise allowed. Also, light selectrons or smuons might be produced in cascade decays, increasing the rate of multi-lepton events which are generally more tightly constrained than purely hadronic events. There are two exceptions to this, however. The constraints on the direct production of $\tilde{\tau}$ leptons are still quite weak, and the $\tau$ tagging efficiency is not large. Allowing the $\tilde{\tau}$ sleptons to be light would therefore probably not make a scenario easier to exclude. On the other hand, as long as the Bino and Wino masses are very large, $\tilde{\tau}$ sleptons would not be produced in stop or gluino decays even if they were light, so allowing light $\tilde{\tau}$ 's would probably not change our conclusions.

The second, and potentially more worrisome, exception is the Bino. If first and second generation squarks are heavy the direct Bino production cross section is very small, so a light Bino would not change the total SUSY production cross section very much. On the other hand a light Bino would allow scenarios where the lighter (higgsino-like) chargino $\tilde{\chi}_{1}^{+}$is heavier than the lighter stop $\tilde{t}_{1}$. If in addition $m_{t}+m_{\tilde{\chi}_{1}^{0}}$ is only slightly smaller than $m_{\tilde{t}_{1}}, \tilde{t}_{1} \rightarrow t+\tilde{\chi}_{1}^{0}$ decays would have a large branching ratio (since this would be the only allowed two-body decay mode of $\tilde{t}_{1}$ ), but a rather poor signature (since $\tilde{t}_{1}$ would then look like a top quark, which has a much larger production cross section and thus contributes a formidable background). This would make searches even for quite light stops difficult, although not impossible [25]. This loophole is not really available in our scenario, since we have $m_{\tilde{\chi}_{1}^{0}} \simeq m_{\tilde{\chi}_{2}^{0}} \simeq m_{\tilde{\chi}_{1}^{+}} \simeq|\mu|<m_{\tilde{t}_{1}}$, where the last inequality follows from our demand that the LSP be the lightest neutralino; hence $m_{t}+m_{\tilde{\chi}_{1}^{0}} \simeq m_{\tilde{t}_{1}}$ would imply that many $\tilde{t}_{1}$ would decay into $b+\tilde{\chi}_{1}^{+}$, which (for $m_{\tilde{\chi}_{1}^{+}} \simeq m_{\tilde{\chi}_{1}^{0}}$ ) looks quite different from a top quark. However, even in a more general model utilizing this loophole requires some finetuning, which arguably is against the spirit of natural supersymmetry. Moreover, at least in standard cosmology a stable Bino-like LSP well separated in mass from all other sparticles would have a much too high cosmological relic density. Avoiding this would require additional finetuning, e.g. by chosing $m_{\tilde{\tau}_{1}} \simeq m_{\tilde{\chi}_{1}^{0}}[26$. In contrast, our spectra, which feature a higgsino-like, relatively light LSP, are cosmologically safe, although in standard cosmology the bulk of the dark matter density would have to be provided by some other particle, e.g. the axion or/and the axino [27.

Finally, one might worry that even if the Bino is too heavy to be produced in stop or gluino decays the 


\begin{tabular}{|c|c|c|}
\hline Parameter & Description & Scanned range \\
\hline$m_{\tilde{Q}_{t}}$ & $3^{\text {rd }}$ generation $S U(2)$ doublet soft breaking squark mass & {$[0.1 \mathrm{TeV}, 1.5 \mathrm{TeV}]$} \\
$m_{\tilde{t}_{R}}$ & $3^{\text {rd }}$ generation $S U(2)$ singlet soft breaking squark mass & {$[0.1 \mathrm{TeV}, 1.5 \mathrm{TeV}]$} \\
$M_{3}$ & Gluino mass parameter & {$[0.1 \mathrm{TeV}, 3.0 \mathrm{TeV}]$} \\
$A_{t}$ & Stop trilinear coupling & {$[-3.0 \mathrm{TeV}, 3.0 \mathrm{TeV}]$} \\
$\mu$ & Higgsino mass parameter & {$[0.1 \mathrm{TeV}, 0.5 \mathrm{TeV}]$} \\
$\tan \beta$ & Ratio of vacuum expectation values & {$[1,20]$} \\
\hline
\end{tabular}

TABLE I. Variable input parameters of our natural SUSY scenario, and the range over which these parameters are scanned. In case of $\mu$ we give the range of the absolute value; negative values of $\mu$ are also sampled. Note that the ranges refer to the running $\overline{\mathrm{DR}}$ parameters, defined at scale $Q=1 \mathrm{TeV}$.

boundaries of the allowed regions might still depend on its mass, since it affects the mass splitting between the higgsino-like states, and hence the amount of visible energy produced in the decays of the heavier states. We show near the end of Sec. IV that this is not the case.

Since in our scenario the only potentially accessible strongly interacting superparticles are gluinos and third generation squarks, the most important production channels are:

$$
p p \rightarrow \tilde{g} \tilde{g}, \quad p p \rightarrow \tilde{t}_{1(2)} \tilde{t}_{1(2)}^{*}, \quad p p \rightarrow \tilde{b}_{1} \tilde{b}_{1}^{*}
$$

where all sparticle production processes can be accompanied by additional initial and/or final state radiation. We have omitted the production of higgsino pairs since the small splitting between the higgsino mass eigenstates, typically $\mathcal{O}(1) \mathrm{GeV}$ in our scenario, make the $\tilde{\chi}_{2}^{0}$ and $\tilde{\chi}_{1}^{ \pm}$decay products too soft for higgsino pair production to be detectable at the LHC. On the other hand, the mass difference is sufficiently large for these decays to be effectively prompt [28]. Hence the chargino and the heavier neutralino mass eigenstate can be treated as missing energy, just like the lightest neutralino. However, the production of a higgsino pair in association with a jet (monojet signature) is negligible at the LHC since the production rate is too small even for Run 2 of the LHC 29.

Depending on the ordering of the states in the spectrum, the decay chains can be relatively complicated. If kinematically allowed, the third generation squarks will dominantly decay via the strong interaction into a gluino and a quark:

$$
\tilde{t}_{a} \rightarrow t \tilde{g}(a=1,2), \quad \tilde{b}_{1} \rightarrow b \tilde{g} .
$$

The squarks can always undergo two-body decay via Yukawa interactions:

$$
\begin{aligned}
& \tilde{t}_{a} \rightarrow t \tilde{\chi}_{l}^{0}(l=1,2), \quad b \tilde{\chi}_{1}^{+}, \\
& \tilde{b}_{1} \rightarrow b \tilde{\chi}_{l}^{0}(l=1,2), \quad t \tilde{\chi}_{1}^{-} .
\end{aligned}
$$

Since the top Yukawa coupling is quite large, the branching ratios for decays (4) will be sizable even if the strong decays (3) are allowed. As shown above, all three higgsino states effectively act as missing energy in our scenario.
In addition, the squarks can have purely bosonic decay modes:

$$
\begin{aligned}
& \tilde{t}_{a} \rightarrow \tilde{b}_{1} W^{+}(a=1,2), \quad \tilde{b}_{1} \rightarrow \tilde{t}_{1} W^{-}, \\
& \tilde{t}_{2} \rightarrow \tilde{t}_{1} Z, \quad \tilde{t}_{2} \rightarrow \tilde{t}_{1} h .
\end{aligned}
$$

Since in our scenario the two-body decay $\tilde{t}_{1} \rightarrow b \tilde{\chi}_{1}^{+}$ is practically always allowed, as noted above, treelevel three- or four-body decays, as well as the loopinduced decay $\tilde{t}_{1} \rightarrow c \tilde{\chi}_{1}^{0}$, which in general can be quite important [30, do not play a role in our scan.

If kinematically allowed, gluinos decay via the treelevel two-body modes

$$
\tilde{g} \rightarrow \tilde{t}_{a} \bar{t}, \quad \tilde{t}_{a}^{*} t(a=1,2), \quad \tilde{b}_{1} \bar{b}, \quad \tilde{b}_{1}^{*} b .
$$

If these decays are kinematically suppressed, treelevel three-body decays via off-shell third generation squarks are possible:

$$
\tilde{g} \rightarrow b \bar{b} \tilde{\chi}_{l}^{0}, \quad t \bar{t} \tilde{\chi}_{0}^{l}(l=1,2) \quad b \bar{t} \tilde{\chi}_{1}^{+}, \quad \bar{b} t \tilde{\chi}_{1}^{-} .
$$

All gluino decay modes in eqs.(8) and (9) give rise to a large $b$-jet multiplicity. For a given mass the gluino, being a color octet fermion, has a much larger cross section than the scalar color triplet third generation squarks. This indicates that searches for final states containing $b$-jets and missing $E_{T}$ will play an important role in probing our natural SUSY scenario. However, if all squarks are much heavier than the gluino, the tree-level two-body modes (8) are closed, and the modes (9) are strongly suppressed by the offshell squark propagator. In this case the loop-induced gluino decays [31, 32.

$$
\tilde{g} \rightarrow g \tilde{\chi}_{l}^{0}(l=1,2)
$$

become important.

\section{NUMERICAL ANALYSIS}

In this section, we first discuss the numerical tools used in this work. Then we describe the generation of model points, summarize theoretical and low energy constraints and describe the framework for testing the model points against LHC data. 


\section{A. Numerical Tools}

The masses and decay branching ratios for each model point in the scan are calculated with SPheno3.3.2 33. For benchmark points with a compressed spectrum 2 , a matched sample of signal events including up to one additional parton (i.e. matching parton-level $2 \rightarrow 2$ and $2 \rightarrow 3$ events) is generated with Madgraph5.1.2 34] interfaced with Pythia 6.4 [35] for showering and hadronization. Otherwise, the entire event generation is handled by Pythia 8.185 [36. We rescale the cross sections with a flat $k$ factor of 1.5. The truth level MC events are passed on to CheckMATE1.2.1 37 39 which is based on the modified fast detector simulation Delphes 3.10 40]. CheckMATE tests if the model point is excluded or not at $95 \%$ confidence level by comparing with published experimental searches at the LHC. Since CheckMATE uses the background estimates provided by the experiments as part of their analyses, no background events had to be generated by us.

\section{B. Scan Procedure}

We have performed a multidimensional scan in the parameters of natural SUSY. To that end, we have randomly generated sets of the free parameters within the ranges shown in Table $\mathbb{1}$, assuming flat probability distributions. The lower bounds on the masses reflect the results of searches at lower energy colliders. In particular, searches at LEP exclude model points where a charged superparticle is lighter than about $100 \mathrm{GeV}$. All other soft breaking parameters are given constant large values, as described in Sec II. The sign of $\mu$ is also chosen randomly with equal a priori probability for positive and negative $\mu$.

The values of these input parameters are passed on to the spectrum generator SPheno, which computes the on-shell masses from the input $\overline{\mathrm{DR}}$ parameters. SPheno also applies theoretical and experimental constraints on the spectrum. All benchmark points must have correct electroweak symmetry breaking, and all sfermions must have positive squared masses. We also demand that the lightest $\mathrm{CP}$-even Higgs boson must have a mass $m_{h}=125 \pm 3 \mathrm{GeV}$, where the range is an estimate of the uncertainty of the calculation. We discard model points where the LSP is not the lightest neutralino. We require the mass difference between the lighter chargino and the LSP to exceed $150 \mathrm{MeV}$, in which case $\tilde{\chi}_{1}^{ \pm}$decays are prompt. The presence of long-lived heavy charged particles in the event would constitute a good signature with little SM background [41.

\footnotetext{
2 The precise definition is given in the next subsection
}

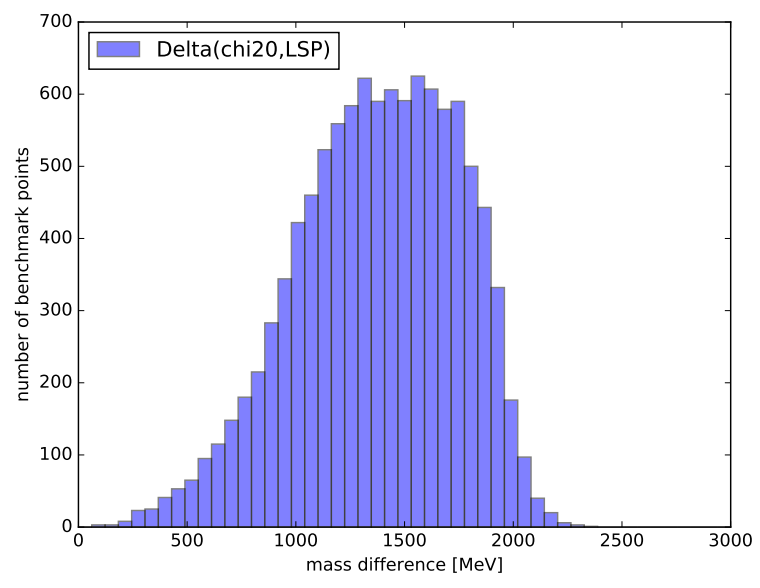

FIG. 1. Distribution of the mass splitting between $\tilde{\chi}_{2}^{0}$ and $\tilde{\chi}_{1}^{0}$ for our model points satisfying the preselection constraints.

We checked that the stop loop contribution to the electroweak $\rho$ parameter 42 is always within the experimental limits, and we expect this to be true for other electroweak precision observables as well, due to the decoupling property of supersymmetric particles. An unpublished combined exclusion limit on the chargino mass by all four LEP collaborations place a lower limit of $103.5 \mathrm{GeV}$. However, for very small mass differences, the limit becomes weaker. We impose a lower mass limit of $100 \mathrm{GeV}$ on the lightest chargino eigenstate from data of the LEP2 run 434 45. We do not explicitly apply any Tevatron limits as a preselection of the benchmark points; recall, however, that we only sample spectra with gluino mass above $100 \mathrm{GeV}$.

We have randomly generated about 22,000 model points satisfying all the preselection cuts. Since the higgsino mass eigenstates are nearly mass degenerate, the NLSP is in general the lighter chargino mass eigenstate or the second lightest neutralino. In Fig. 1 we show the mass splitting between the LSP and the second lightest neutralino. For a mass splitting larger than the pion mass, the decay of the heavier neutralino is effectively prompt. However, this figure also shows that the visible $\tilde{\chi}_{2}^{0}$ decay products will almost always be too soft to be observed at the LHC.

In Fig. 2 we show histograms of the scalar particle masses. We see a significant mass splitting between the lighter and the heavier stop mass eigenstates. For relatively light $\tilde{t}_{1}$, a rather heavy $\tilde{t}_{2}$ is required to obtain a sufficiently large value of $m_{h}$ in the MSSM; the smallest value of $m_{\tilde{t}_{2}}$ among the 22,000 model points is just under $800 \mathrm{GeV}$. Since even within simplified models current lower bounds on third generation sparticle masses do not exceed $700 \mathrm{GeV}, \tilde{t}_{2}$ pair production by itself does not lead to significant constraints on our model points. Thus we will be sensitive to the bosonic decays $\tilde{t}_{2}$ as described in Eq. (7) mostly if $\tilde{t}_{2}$ is pro- 


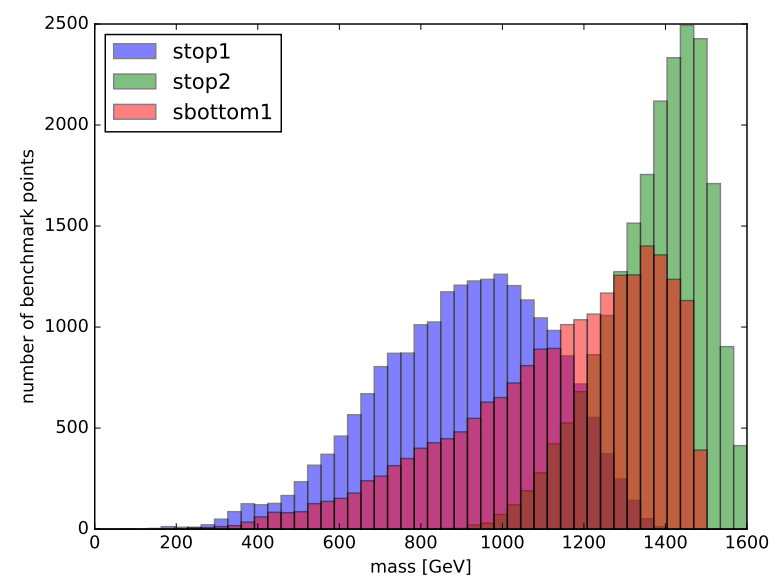

FIG. 2. Distributions of the masses of the third generation squarks $\tilde{t}_{1}, \tilde{t}_{2}$ and $\tilde{b}_{1}$ for our model points satisfying the preselection constraints.

duced in gluino decays.

As expected, the mass of the lighter sbottom covers a large range. If the lighter stop mass eigenstate is dominantly a $S U(2)$ doublet, a light sbottom mass eigenstate with similar mass will also emerge in the sparticle spectrum. Bosonic $\tilde{b}_{1} \rightarrow \tilde{t}_{1} W^{ \pm}$decays, see Eq. (6), can be important as long as $\tilde{t}_{1}$ has a significant $S U(2)$ doublet component. This could lead to observable signals even if direct stop pair production is not observable because the mass splitting to the LSP is too small. Finally, recall that we fixed the $\tilde{b}_{R}$ mass to a very large value, so $\tilde{b}_{R}$ production does not play any role in our scan.

Fig. 3 shows the gluino mass distribution. It is basically flat above $500 \mathrm{GeV}$; this is not unexpected, since the soft breaking $(\overline{\mathrm{DR}})$ gluino mass is one of the input parameters that is randomly sampled from a flat distribution. The distribution falls off below $500 \mathrm{GeV}$ since we discard points with $m_{\tilde{g}}<m_{\tilde{\chi}_{1}^{0}} \simeq|\mu|$, and we require $|\mu|<500 \mathrm{GeV}$. In simplified models gluino pair production followed by gluino decay into third generation (s)quarks can be probed by published LHC searches for gluino masses up to $1.3 \mathrm{TeV}$. We also sampled model points with significantly heavier gluinos since in principle combinations of gluino and third generation squark pair production might exclude model points where squark and gluino production by itself satisfies all constraints. Moreover, we wanted to have a statistically meaningful sample where effectively only the third generation squarks could contribute to signatures at run 1 of the LHC.

We saw in the last section that natural supersymmetry scenarios cover a large number of final state topologies. The relative importance of these topologies depends on the details of the particle spectrum, e. g. on the mass ordering and the mixing between the stop current eigenstates. Fortunately, both LHC

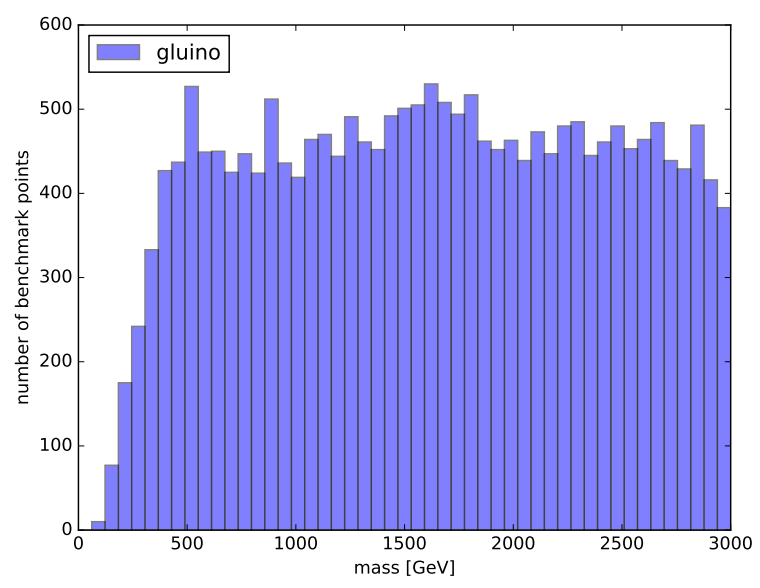

FIG. 3. Distributions of the masses of the gluino for our model points satisfying the preselection constraints.

experiments ATLAS and CMS have covered a large number of final states relevant for the production of stops, sbottoms as well as gluinos. Moreover, both experiments have performed powerful "inclusive" SUSY searches, targeting final states with (generally untagged) jets and a large amount of missing $E_{T}$, in some cases also requiring the presence of charged leptons. These inclusive searches were used primarily to derive limits on the parameter space of constrained supersymmetric models, but they can also be sensitive to our natural SUSY scenario. By considering all these searches together we expect to obtain improved limits on the parameter space.

The relevant searches implemented in CheckMATE are listed in Table II. The left column gives an identifier for the given search; published results are identified by their arXiv number, while results from conference proceedings are identified with their ATLAS or CMS internal number. The second column of this Table show the final state signature for which the given analysis is optimized. The third column gives the total integrated luminosity used in that analysis. More details about these twelve experimental searches are given in Appendix A

It should be mentioned that the preponderance of ATLAS searches is simply due to the historical accident that CheckMATE currently has implemented many more ATLAS than CMS analyses. Generally ATLAS and CMS searches for a given final state show similar sensitivity. Since we do not statistically combine different searches, adding CMS searches for the final states also searched for by ATLAS would not change our results very much. We do include a couple of CMS searches that do not have a close ATLAS equivalent.

In order to predict the number of signal events for all signal regions of the various analyses several simulation steps are needed. We first generate 5, 000 truth level Monte Carlo (MC) events for each benchmark 


\begin{tabular}{|c|c|c|}
\hline Reference & Final State & $\mathcal{L}\left[\mathrm{fb}^{-1}\right]$ \\
\hline 1308.2631 (ATLAS) 51] & $0 \ell+2 b$ jets $+\mathbb{E}_{T}$ & 20.1 \\
\hline 1403.4853 (ATLAS) 52 & $2 \ell+\mathbb{E}_{T}$ & 20.3 \\
\hline 1404.2500 (ATLAS) 54 & SS $2 \ell$ or $3 \ell$ & 20.3 \\
\hline 1407.0583 (ATLAS) [55] & $1 \ell+(b)$ jets $+\mathbb{E}_{T}$ & 20.0 \\
\hline 1407.0608 (ATLAS) [56] & monojet $+\mathbb{E}_{T}$ & 20.3 \\
\hline 1303.2985 (CMS) [57] & $\alpha_{T}+b$ jets & 11.7 \\
\hline ATLAS-CONF-2012-104 [58] & $1 \ell+\geq 4$ jets $+\mathbb{E}_{T}$ & 5.8 \\
\hline ATLAS-CONF-2013-024 [59] & $0 \ell+6(2 b)$ jets $+\mathbb{E}_{T}$ & 20.5 \\
\hline ATLAS-CONF-2013-047 60 & $0 \ell+2-6$ jets $+\mathbb{E}_{T}$ & 20.3 \\
\hline ATLAS-CONF-2013-061 61 & $0-1 \ell+\geq 3 b \mathrm{jets}+\mathbb{E}_{T} \mid$ & 20.1 \\
\hline ATLAS-CONF-2013-062 62 & $1-2 \ell+3-6$ jets $+\mathbb{E}_{T}$ & 20.0 \\
\hline CMS-SUS-13-016 63. & OS $2 \ell+\geq 3 b$ jets & 19.7 \\
\hline
\end{tabular}

TABLE II. The experimental analyses used in our study. The ${ }^{*} \mathrm{CONF}^{*}$ papers are only published as conference proceedings, the others are given by their arXiv number. The middle column denotes the final state for which the analysis is optimized, and the third column shows the total integrated luminosity employed in this analysis.

point, including all processes given in Eq. (2). The corresponding total cross section before cuts is also computed at this step; as noted above, we scale up the leading order cross section by a universal " $k$-factor" of 1.5 .

For strongly interacting sparticles with relatively small mass splitting to the LSP, leading to rather soft visible decay products, an accurate treatment of additional radiation is important; in the extreme case of very small mass splitting this can give rise to a monojet signature 46, which has been searched for in one of the analyses we consider [56]. We therefore describe the pair production of third generation squarks or gluinos with mass less than $300 \mathrm{GeV}$ above the LSP mass by matching event samples with two and three partons in the final state, using the parton-jet MLM matching algorithm described in Ref. [47. The numerical matching is performed with Madgraph interfaced with the shower generator Pythia6.4, where we applied a $p_{T}$ sorted parton shower and hadronization is switched on. We generate 50,000 MadGraph events for each of these model points; this tenfold increase overcompensates the fact that some events are removed in the matching process. In order to reduce the required computational effort, the production of superparticles with larger mass splitting to the LSP is directly handled by Pythia8.185 without matching. In some cases, Pythia was not able to hadronize final states from sparticle decays with very small splitting and we removed those model points.

The truth level MC events are then further processed with the tool CheckMATE. It consists of a simulation of the detector response with a modified Delphes where the settings have been retuned to mimic the responses of the ATLAS detector. In particular, an

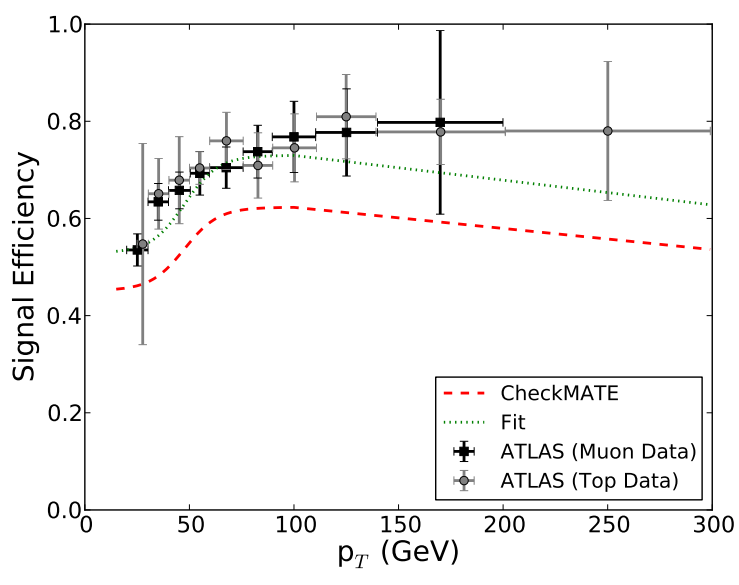

FIG. 4. The data points show $b$-tagging efficiencies as determined by the ATLAS collaboration from two different analyses [48, 49], for one specific working point. The dotted line is a fit to these data points, and the red dashed curve shows the actual CheckMATE implementation, which has been scaled down by a factor 0.85 as described in the text.

accurate description of the $b$-tagging efficiency is of crucial importance since, as shown in Table II many third generation searches rely on $b$-tagging. The tagging efficiency measured by ATLAS and its implementation in CheckMATE are shown in Fig. 4 as a function of the $p_{T}$ of the $b$-jet. Here the parameters of the tagging algorithm have been chosen such that the overall $b$-tagging efficiency for $t \bar{t}$ events is $70 \%$. Two different data sets have been used to derive the fit shown in this figure, with different sensitivities at small and large momenta [48, 49]. Moreover, the overall normalization has been reduced by $15 \%$ in order to obtain better agreement between the CheckMATE implementation of ATLAS and CMS new physics searches and the actual experimental results. This could be due to the final states in new physics searches typically being more complicated than the ones used to determine the $b$-tagging efficiency shown in Fig. 4 .

The reconstructed detector level objects, as well as the total cross sections computed earlier, are then passed on to the analysis module of CheckMATE. All studies listed in Table II have been implemented in CheckMATE and have been carefully validated against the results published by the experiments. CheckMATE typically reproduces the total cut efficiency given by the experiments with an accuracy of $10 \%$ or better. More details on the validation of all implemented experimental searches can be found in the CheckMATE manual and web page [37, 38.

We check each model point against all the analyses given in Table II. Note that each of these analyses defines several "signal regions", defined by sets of kinematic cuts. Out of these many regions, CheckMATE 
finds the one with the largest expected exclusion potential; this is computed from the background determined by the experimenters and its error, as well as the signal cross section times cut efficiency for this particular signal region, and is independent of the actually observed number of events in this signal region. Finally, for the signal region selected in this manner, CheckMATE compares the sum of the background and the predicted signal with the actual experimental observation and determines if the model point is excluded at the $95 \%$ C.L., using the so-called $\mathrm{CL}_{S}$ method [50]. More specifically, it computes the parameter

$$
r \equiv \frac{S-1.96 \cdot \Delta S}{S_{\mathrm{exp}}^{95}},
$$

where $S$ is the number of signal events, $\Delta S$ denotes its theoretical uncertainty, and and $S_{\text {exp. }}^{95}$ is the experimentally determined $95 \%$ confidence level limit on the signal. We only include the error from the limited statistics of our Monte Carlo simulation, i.e. $\Delta S=\sqrt{S}$. The actual (as opposed to expected) value of $r$ is only computed for the "optimal" signal region defined above, in order to avoid spurious exclusions due to downward fluctuations in the data; since the analyses we employ define well over a hundred signal regions, we expect several $2 \sigma$ fluctuations to have occurred in these data. In order to keep the statistical analysis simple and transparent, CheckMATE does not statistically combine signal regions of a particular analysis, nor does it combine different analyses. CheckMATE considers a model to be excluded at $95 \%$ c.l. level if $r$ defined in eq.(11) exceeds 1. According to this strict definition, Monte Carlo fluctuations would decide whether scenarios with $r \approx 1$ are considered excluded or allowed. We therefore increase the event sample to 50,000 whenever the original assessment of $r$ gave a value between $2 / 3$ and $3 / 2$. Moreover, we conservatively consider a model point to be (definitely) excluded only if our final estimate gives $r \geq 1.5$, while points with $r \leq 2 / 3$ are considered (definitely) allowed. Model points with $2 / 3<r<3 / 2$ are thus indeterminate. This can be considered to be a (probably rather conservative) simple method to include theoretical uncertainties on the predicted signal strengths.

\section{LIMITS ON GLUINO AND THIRD GENERATION SCALAR MASSES}

We are now ready to present numerical results of our scan. We first make some general remarks and then show the distribution of allowed and excluded points in the planes spanned by two of the three most important model parameters, which are the masses of the gluino, of the lighter stop, and of the LSP. We also delineate completely excluded as well as completely allowed regions of parameter space. Finally, we discuss the properties of model points which evade current collider searches.

As previously described, we have randomly generated 22,000 model points. In the majority of these points all superparticles are beyond the reach of LHC run 1 , and thus these models points are still allowed. However, about $25 \%$ of all model points are ruled out by the experimental searches we consider $(r>1.5)$ and another $6.6 \%$ are indeterminate $(2 / 3<r<3 / 2)$.

Table III compiles statistics about the twelve considered analyses. Columns 3 through 6 give the fractions of model points for which a given analysis is the most sensitive one. This is shown with respect to the entire set of model points (col. 3), as well as specifying to clearly excluded (col. 4), ambiguous (col. 5) and clearly allowed model points (col. 6).

We see that the ATLAS search [61] for final states with missing transverse momentum and at least three $b$ jets performs best among the clearly excluded and ambiguous points. This is not surprising since we expect a large number of $b$ jets from direct gluino pair production with subsequent decay into third generation sparticles. The ATLAS inclusive multijet plus missing $E_{T}$ search with a charged lepton veto 60 also plays an important role in constraining natural SUSY even though the study is not optimized for this scenario. In fact, we see that this search offers the best sensitivity for clearly allowed points. This indicates that in future inclusive SUSY searches might be more important in further constraining the currently still allowed parameter space of natural SUSY than dedicated searches for third generation squarks. This is related to our upper bound $|\mu|<500 \mathrm{GeV}$, which ensures that model points where all strongly interacting sparticles are well beyond current sensitivity limits will have large mass splitting to the LSP. This implies good sensitivity for the inclusive search, without having to pay the price in efficiency that is required by multiple $b$-tags.

While either of these two analyses performs best in nearly two third of the excluded model points, a total of six further searches sometimes have the best sensitivity. This shows the importance of including a large set of experimental searches when constraining the parameter space even of our relatively simple implementation of natural supersymmetry. In particular, the inclusive CMS $\alpha_{T}$ analysis [57] classifying events according to their $b$ jet multiplicity performs quite well despite the fact that the data sample only corresponds to an integrated luminosity of 11.7 inverse femtobarn. The ATLAS single lepton search [55] also does a reasonably good job. In our case decays of the stop into $\tilde{t}_{1} \rightarrow t \tilde{\chi}_{1}^{0}$, or three-body gluino decays including at least one top quark in the final state, will result in many events with one isolated lepton.

On the other hand, we see that searches that require two or more charged leptons never have the best sensitivity to our model points. The rate for two lepton final states is heavily suppressed by the leptonic branch- 


\begin{tabular}{|c|c|c|c|c|c|c|}
\hline \multirow[t]{2}{*}{ Experiment } & \multirow[t]{2}{*}{ Final State } & \multicolumn{4}{|c|}{ Best Sensitivity } & \multirow[t]{2}{*}{ Excludes } \\
\hline & & all & excluded & ambiguous & allowed & \\
\hline ATLAS 61 & $0-1 \ell+\geq 3 b$ jets $+\mathbb{E}_{T}$ & 0.22 & 0.37 & 0.56 & 0.13 & 0.57 \\
\hline ATLAS 60 & $0 \ell+2-6$ jets $+\mathbb{E}_{T}$ & 0.37 & 0.25 & 0.056 & 0.44 & 0.69 \\
\hline CMS [57] & $\alpha_{T}+b$ jets & 0.088 & 0.11 & 0.14 & 0.075 & 0.66 \\
\hline ATLAS 59. & $0 \ell+6(2 b)$ jets $+\mathbb{E}_{T}$ & 0.044 & 0.12 & 0.041 & 0.016 & 0.58 \\
\hline ATLAS 55 & $1 \ell+(b)$ jets $+\mathbb{E}_{T}$ & 0.14 & 0.078 & 0.10 & 0.16 & 0.45 \\
\hline ATLAS 56] & monojet $+\mathbb{E}_{T}$ & 0.013 & 0.042 & 0.018 & 0.002 & 0.23 \\
\hline ATLAS 51. & $0 \ell+2 b$ jets $+\mathbb{E}_{T}$ & 0.10 & 0.019 & 0.085 & 0.13 & 0.051 \\
\hline ATLAS 62 & $1-2 \ell+3-6$ jets $+\mathscr{E}_{T}$ & 0.024 & 0.002 & 0.001 & 0.034 & 0.50 \\
\hline ATLAS 54 & $\mathrm{SS} 2 \ell$ or $3 \ell$ & 0.0 & 0.0 & 0.0 & 0.0 & 0.070 \\
\hline ATLAS 58 & $1 \ell+\geq 4$ jets $+\mathbb{E}_{T}$ & 0.0 & 0.0 & 0.0 & 0.0 & 0.12 \\
\hline CMS 63. & OS $2 \ell+\geq 3 b$ jets & 0.0 & 0.0 & 0.0 & 0.0 & 0.043 \\
\hline ATLAS 52 & $2 \ell+\mathbb{E}_{T}$ & 0.0 & 0.0 & 0.0 & 0.0 & 0.0 \\
\hline
\end{tabular}

TABLE III. Statistical information about the sensitivity of the twelve analyses with respect to our natural SUSY scenarios. The first column gives the name of the experiment and the references. The corresponding final state is given in the second columns. Columns three through six give the fraction of model points for which this analysis is the most sensitive one; column 3 is for the entire set of model points, while the next three columns only include model points with $r>1.5,2 / 3<$ $r<3 / 2$ and $r<2 / 3$, respectively. The last column gives the fraction of all excluded model points that are (also) excluded by this particular analysis, i.e. where this analysis has $r>1.5$.

ing ratio. This might change, however, if we allowed sleptons to be relatively light, which would e.g. be required if loops with supersymmetric particles were to explain the $\sim 3 \sigma$ deviation in the anomalous magnetic moment of the muon. It should be noted that the multivariate analysis targeting the decay mode $\tilde{t}_{1} \rightarrow t \tilde{\chi}_{1}^{0}$ in 52 is not implemented in this work, since CheckMATE currently only allows to implement cut-based analyses; the other signal regions in [52] are optimized for stop decays into charginos with leptonic chargino decays and thus dilepton final states from $\tilde{t}_{1} \rightarrow t \tilde{\chi}_{1}^{0}$ decays are frequently missed. Same sign tops, leading to events with two same sign leptons, can be produced in the decays of the gluino. An analogous signature can also arise from gluino mediated sbottom production with the subsequent decay $\tilde{b}_{1} \rightarrow t \tilde{\chi}_{1}^{ \pm}$. Events with same sign leptons have a very small SM background. However, again due to the small cumulative branching ratio the SS dilepton search does not perform as well as the other searches.

The last column in Table III shows the fraction of clearly excluded model points (i.e. points with $r>1.5$ ) that are excluded by this analysis, i.e. where the best signal region of a given analysis has $r>1.5$. The entry in this column is obviously larger than that in the second column, which only counts the fraction of model points for which this particular analysis performs best. The sum of the entries in this column is significantly larger than 1 , showing that many disallowed points are in fact excluded by several independent analyses. Even the searches for final states with two or more leptons, which never offer the best sensitivity as we just saw, do exclude some of our points, i.e. they help to increase the confidence level with which these points can be excluded; the only exception is the ATLAS search of ref. [52], which does not exclude any of our model points.

Now we want to discuss where in the overall parameter space the experimentally excluded as well as the clearly allowed model points are situated. To this end we show in Figs. 5 to 8 the planes spanned by two of the most relevant masses, which are the masses of the gluino, of the lighter stop eigenstate, and of the LSP. In these figures clearly excluded points (with $r>1.5)$ are marked in red, and clearly allowed points (with $r<2 / 3$ ) are shown in green. Ambiguous points are not shown at all, in order to better illustrate the separation between allowed and excluded regions of parameter space.

Fig. 5 shows the $\tilde{t}_{1}$ and $\tilde{g}$ mass plane. Not surprisingly, points where both these masses are large cannot be excluded, simply because the total cross section for the production of superparticles was too small at run 1 of the LHC. Specifically, we see that no scenarios that satisfy $m_{\tilde{t}_{1}}>580 \mathrm{GeV}$ and $m_{\tilde{g}}>1070 \mathrm{GeV}$ are excluded. Similarly, all points with $m_{\tilde{t}_{1}}>660 \mathrm{GeV}$ and $m_{\tilde{g}}>1180 \mathrm{GeV}$ are clearly allowed. The gap at $m_{\tilde{g}} \simeq 1100 \mathrm{GeV}$ is due to the ambiguous points. The fact that a clear gap appears indicates that our definitions of allowed and excluded points are indeed conservative, i.e. few if any points get labeled allowed or excluded just due to Monte Carlo fluctuation; the latter only shift points between the clearly allowed and ambiguous categories, as well as between the clearly excluded and ambiguous categories.

While Fig. 5 allows to define a region of the $m_{\tilde{g}}, m_{\tilde{t}_{1}}$ plane where all points are allowed, and a slightly larger region where no points are excluded, finding a region 


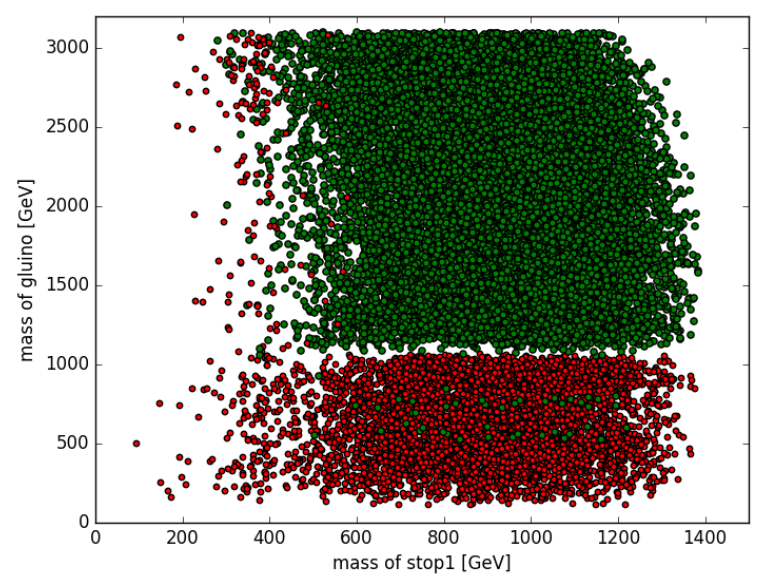

FIG. 5. Models points in the stop and gluino mass plane. Model points clearly passing all constraints are shown in green, while clearly excluded points are shown in red.

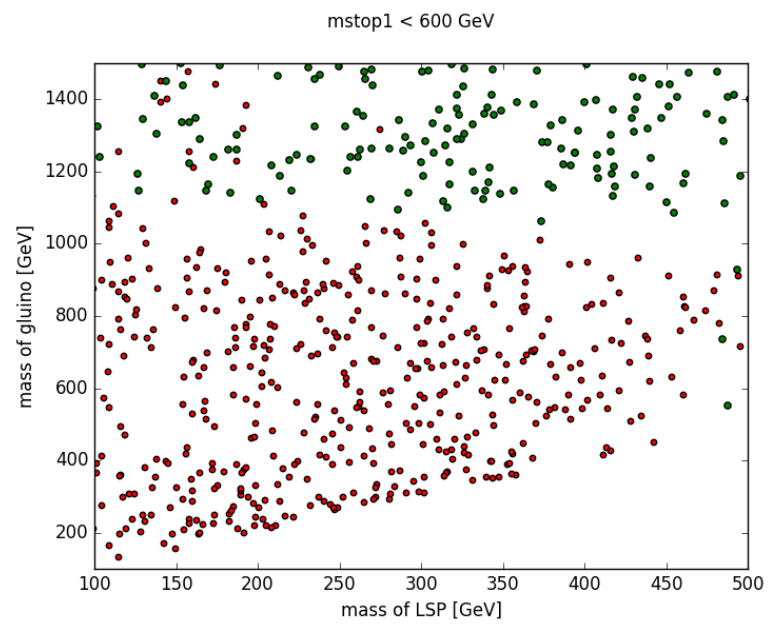

FIG. 6. Model points with the lighter stop mass less than $600 \mathrm{GeV}$ in the LSP and gluino mass plane. The notation is as in Fig. 5

of this plane where all points are excluded is not so easy. For example, we see that some points with $m_{\tilde{g}}$ well above $1.2 \mathrm{TeV}$ and $m_{\tilde{t}_{1}}<600 \mathrm{GeV}$ are excluded, but other points with similar combination of gluino and lighter stop mass are allowed. Similarly, there are a few allowed points with gluino mass well below 1 $\mathrm{TeV}$.

The reason for this intermingling of allowed and excluded points is that Fig. 5 does not distinguish between different values of the LSP mass. The mass gap between the LSP and the directly produced strongly interacting superparticles largely determines the amount of visible energy, and of missing transverse momentum, in the event. In Fig. 6 we therefore present the allowed (excluded) model points in green (red) in the LSP and gluino mass plane while demanding that the lighter stop mass eigenstate is lighter

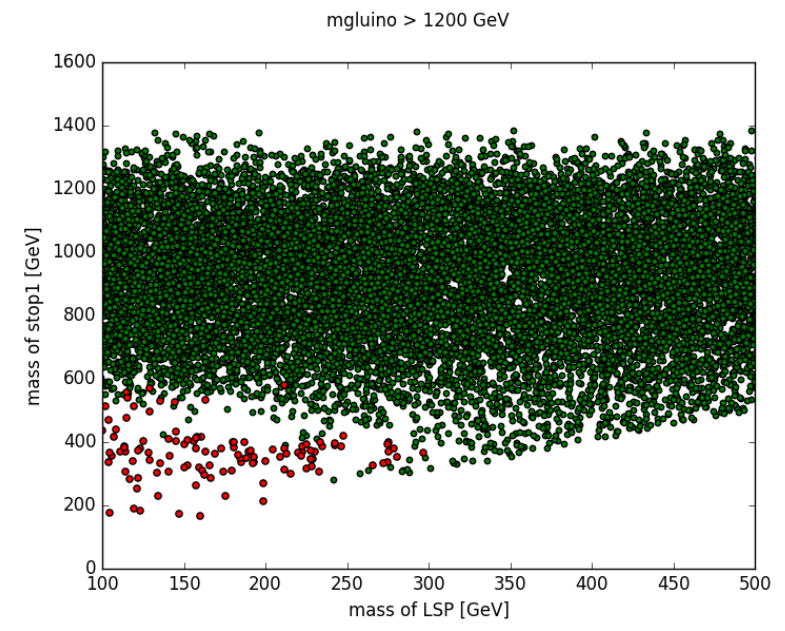

FIG. 7. Models points with the gluino mass greater than $1200 \mathrm{GeV}$ in the LSP and stop mass plane. The notation is as in Fig. 5

than $600 \mathrm{GeV}$. We see that now no model points with gluinos masses less than $1000 \mathrm{GeV}$ and LSP mass below $480 \mathrm{GeV}$ are clearly allowed. Only a few model points with $m_{\tilde{g}}<1 \mathrm{TeV}$ are allowed, which have LSP mass near the upper limit of our scan, leading to a relatively small amount of visible energy in the events. Similarly, all points with $m_{\tilde{t}_{1}}<600 \mathrm{GeV}, m_{\tilde{g}}<950$ $\mathrm{GeV}$ and $m_{\tilde{\chi}_{1}^{0}}<400 \mathrm{GeV}$ are clearly excluded.

A certain number of model points with heavy gluino, $m_{\tilde{g}} \geq 1100 \mathrm{GeV}$, are also excluded. It is clear from our discussion of Fig. 5 that in these cases the exclusion is mostly due to direct stop and, perhaps, sbottom pair production. However, since for equal masses the $\tilde{t}_{1}$ and $\tilde{b}_{1}$ pair production cross sections are far smaller than the $\tilde{g}$ pair production cross section, direct squark pair production only excludes a relatively small region of parameter space. In particular, we see from Fig. 6 that even for relatively light $\tilde{t}_{1}, m_{\tilde{t}_{1}}<600 \mathrm{GeV}$, no points with $m_{\tilde{g}}>1100 \mathrm{GeV}$ and $m_{\tilde{\chi}_{1}^{0}}>300 \mathrm{GeV}$ are clearly excluded.

The limited scope of searches for direct pair production of third generation squarks is further illustrated in Fig. 7. which shows all points with $m_{\tilde{g}}>1.2 \mathrm{TeV}$ in the $\tilde{t}_{1}$ and LSP mass plane. We see again that no point with heavy gluino and $m_{\tilde{t}_{1}}>600 \mathrm{GeV}$ is currently excluded. Even for LSP masses near the lower limit allowed by LEP searches, there are allowed points with $m_{\tilde{t}_{1}} \lesssim 450 \mathrm{GeV}$, and ambiguous points with $m_{\tilde{t}_{1}} \lesssim 350$ $\mathrm{GeV}$. Only the region $m_{\tilde{t}_{1}}<300 \mathrm{GeV}, m_{\tilde{\chi}_{1}^{0}}<210 \mathrm{GeV}$ is completely excluded by these searches.

Fig. 8 shows the plane spanned by the gluino and LSP masses, and only includes points where squarks are too heavy for direct squark pair production to exclude the scenario, i.e. $m_{\tilde{t}_{1}} \geq 600 \mathrm{GeV}$. The limit is thus essentially determined by the production of gluino pairs. Recall that in natural SUSY, gluinos quite often decay into third generation squarks; in par- 


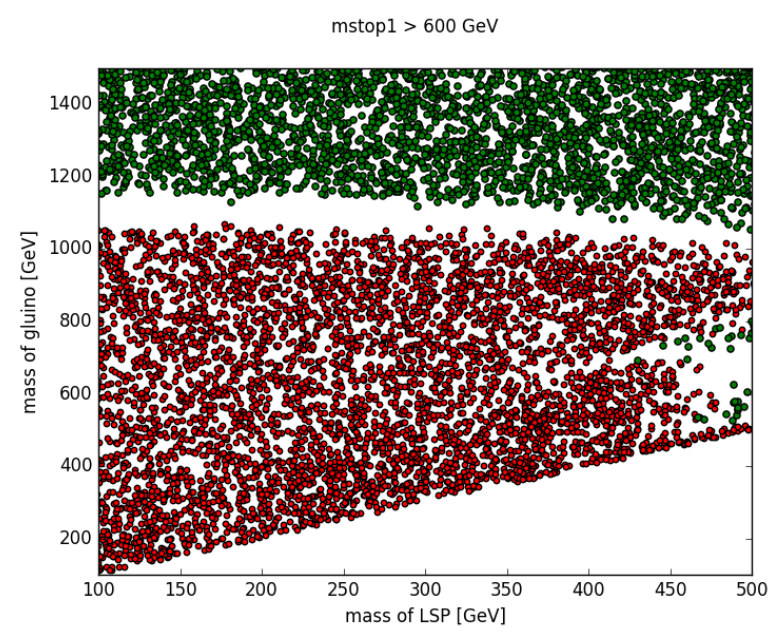

FIG. 8. Models points with $m_{\tilde{t}_{1}}>600 \mathrm{GeV}$ in the LSP and gluino mass plane. The notation is as in Fig. 5 .

ticular, final states containing at least one $t \bar{t}$ pair are quite common also in many of these scenarios.

We observe a clear separation between an entirely allowed and a mostly excluded region. Again, the gap arises because we do not show ambiguous model points which are neither clearly excluded nor clearly allowed. The lower edge of the allowed region is reduced by about $100 \mathrm{GeV}$ as the LSP mass increases from its lower bound near $100 \mathrm{GeV}$ to the maximal value near $500 \mathrm{GeV}$ considered in our definition of natural supersymmetry; evidently the increased LSP mass reduces the visible energy, and hence the efficiency of the cuts of the most sensitive analyses. However, even for $m_{\tilde{\chi}_{1}^{0}}$ near its upper bound the mass splitting to the gluino exceeds $500 \mathrm{GeV}$ in this large clearly allowed region, so we are not yet dealing with a compressed spectrum.

This is different for the small island of allowed model points near the right boundary of Fig. 8, with LSP mass above $430 \mathrm{GeV}$ and gluino mass below $800 \mathrm{GeV}$. Fixing the LSP mass near $500 \mathrm{GeV}$, we see that reducing the gluino mass below $1000 \mathrm{GeV}$ in many cases reduces the signal after cuts, i.e. the increase of the total gluino pair production cross section is overcompensated by the reduction of the cut efficiencies of the most sensitive analyses. Somewhat surprisingly this allowed region does not extend all the way down to the line $m_{\tilde{\chi}_{1}^{0}}=m_{\tilde{g}}$, which are excluded by monojet searches in ref. [56] even for the highest LSP masses in our scan. As a result, the region where no points are excluded is quite small; for instance, no points with $m_{\tilde{t}_{1}}>600 \mathrm{GeV}, m_{\tilde{g}} \in[600,760] \mathrm{GeV}$ and $m_{\tilde{\chi}_{1}^{0}}>470$ $\mathrm{GeV}$ can be excluded. However, even in this narrow range of gluino masses, there are allowed points with LSP mass down to about $430 \mathrm{GeV}$, and ambiguous points up to the highest LSP mass in our scan. The reason is that the gluino branching ratios still depend on the masses of third generation squarks, even if the latter are too heavy for their pair production to con- tribute significantly to the total SUSY cross section.

The various excluded, not excluded, and clearly allowed regions are summarized in Table IV. Here an "excluded (allowed) region" is a region in parameter space in which all model points are excluded (allowed), while a "not excluded" region is a region where no model points are excluded; the latter regions include ambiguous points.

\begin{tabular}{|l|l|}
\hline Type & Boundaries \\
\hline Allowed & $m_{\tilde{t}_{1}}>660 \mathrm{GeV}$ and $m_{\tilde{g}}>1180 \mathrm{GeV}$ \\
& $m_{\tilde{g}}>1150 \mathrm{GeV}$ and $m_{\tilde{\chi}_{1}^{0}}>370 \mathrm{GeV}$ \\
\hline Not & $\begin{array}{l}m_{\tilde{t}_{1}}>580 \mathrm{GeV} \text { and } m_{\tilde{g}}>1070 \mathrm{GeV} \\
\text { excluded } \\
m_{\tilde{g}}>1060 \mathrm{GeV} \text { and } m_{\tilde{\chi}_{1}^{0}}>300 \mathrm{GeV} \\
m_{\tilde{t}_{1}}>550 \mathrm{GeV}, m_{\tilde{\chi}_{1}^{0}}>470 \mathrm{GeV} \\
\text { and } m_{\tilde{g}} \in[600 \mathrm{GeV}, 760 \mathrm{GeV}]\end{array}$ \\
\hline Excluded & $\begin{array}{l}m_{\tilde{t}_{1}}<230 \mathrm{GeV} \text { or } m_{\tilde{g}}<440 \mathrm{GeV} \\
m_{\tilde{g}}<990 \mathrm{GeV} \text { and } m_{\tilde{\chi}_{1}^{0}}<340 \mathrm{GeV} \\
m_{\tilde{g}}<1040 \mathrm{GeV} \text { and } m_{\tilde{\chi}_{1}^{0}}<200 \mathrm{GeV} \\
m_{\tilde{t}_{1}}<300 \mathrm{GeV} \text { and } m_{\tilde{\chi}_{1}^{0}}<210 \mathrm{GeV}\end{array}$ \\
\hline
\end{tabular}

TABLE IV. List of allowed, not excluded and excluded regions. In the allowed regions, all model points have $r<$ $2 / 3$; in the excluded regions, all model points have $r>1.5$; and in the not excluded regions, all model points have $r<$ 1.5

Note that there are points that are neither in one of the "excluded" nor in one of the "not excluded" regions listed in Table IV] The reason is that this table defines regions only based on the values of three parameters: the masses of the gluino, of the lighter stop, and of the LSP. While these are the most important parameters deciding whether a model point is excluded or not, they are not the only ones. For example, the mass of the lighter sbottom is also relevant. In our scan we always have $m_{\tilde{b}_{1}}>m_{\tilde{t}_{1}}$, but the mass difference is typically quite small if $\tilde{t}_{1}$ is mostly an $S U(2)$ doublet, which requires $m_{\tilde{Q}_{t}}<m_{\tilde{t}_{R}}$. The presence of $\tilde{b}_{1}$ only slightly above $\tilde{t}_{1}$ obviously increases the total squark pair production cross section. Moreover, $\tilde{b}_{1}$ and $\tilde{t}_{1}$ pair production often yield essentially the same final state, if $\tilde{t}_{1} \rightarrow t \tilde{\chi}_{1,2}^{0}$ and $\tilde{b}_{1} \rightarrow t \tilde{\chi}_{1}^{-}$; if kinematically allowed, these are typically the most important decay modes if $\tilde{t}_{1}$ is mostly an $S U(2)$ doublet. In contrast, a mostly $S U(2)$ singlet $\tilde{t}_{1}$ prefers to decay into $b \tilde{\chi}_{1}^{+}$ even if the decays into $t \tilde{\chi}_{1,2}^{0}$ are kinematically allowed. The reason is that for the relevant case of higgsino-like lighter chargino, the $\tilde{t}_{R} b \tilde{\chi}_{1}^{ \pm}$coupling is proportional to the top Yukawa coupling, while the $\tilde{t}_{L} b \tilde{\chi}_{1}^{ \pm}$coupling is proportional to the much smaller bottom Yukawa coupling. This difference in $\tilde{t}_{1}$ decay modes also affects the $r$ value of a model point. 
The upshot of this discussion is that some combinations of $m_{\tilde{g}}, m_{\tilde{t}_{1}}$ and $m_{\tilde{\chi}_{1}^{0}}$ can be allowed, excluded or ambiguous depending on the values of the other parameters. However, Table IV] shows that over much of the parameter space these three parameters suffice to determine the fate of a model point.

In some cases strong dependence of the cut efficiency on kinematic quantities, together with fluctuations of the numbers of events actually observed in certain search regions, also leads to quite large differences in $r$ between points that are quite close in parameter space. For instance, we found a pair of model points, p74 and p11081 in our scan, with quite similar spectra and decay branching ratios, yet their $r$ values differ by a factor of two. In both cases the gluino is so heavy that its production can be ignored, while both $\tilde{t}_{1}$ and $\tilde{b}_{1}$ lie at or below $600 \mathrm{GeV}$, while the LSP mass is relatively light. As explained above, both $\tilde{t}_{1}$ and $\tilde{b}_{1}$ then decay predominantly into a top quark and a higgsinolike neutralino or chargino; recall that the two heavier higgsino-like states effectively behave the same way as the LSP, as far as LHC signatures are concerned. Point p11081 has about $9 \%$ heavier squarks, and about $40 \%$ heavier higgsinos, such that the energy of the top in the rest mass of the decaying $\tilde{t}_{1}$ is nearly the same in both cases. Due to the larger squark masses this scenario has nearly two times smaller squark production cross section than p74, yet it yields a two times larger value of $r$; as a result, p11081 is clearly excluded, while p74 is ambiguous.

We found that the small kinematic differences between the two scenarios lead to significantly different efficiencies in the ATLAS search for a hadronically decaying top pair plus missing $E_{T}$ [59. As a result, the signal region expected to be most sensitive to p11081 is from this search, whereas for p74 it is from [55, which searches for final states with one lepton, two jets and missing $E_{T}$. Moreover, the relevant signal region in 59 contains fewer events than expected from backgrounds, while the relevant signal region in 55 contains somewhat more events than predicted in the background-only hypothesis; these differences are likely due to fluctuations. As a result, the actual $r$ value is higher than expected for p11081, but lower than expected in p74.3 This, together with the strong dependence of the cut sensitivity of the relevant analysis in 59, leads to the counter-intuitive outcome that only the heavier spectrum is excluded.

\footnotetext{
3 The LHC experiments use the $S_{95}$ method 64 of setting limits. This ensures that an under-fluctuation by more than two standard deviations, which formally rules out the SM at the $95 \%$ c.l. (without "look elsewhere" effect), does not exclude all scenarios where the expected number of events is larger than in the SM. This is essential, since given the large number of signal regions included in the analysis, it is highly likely that some fluctuate down by more than two standard deviations. However, such a downward fluctuation still does increase the $r$ value even in the $S_{95}$ method, as indeed it should.
}

Finally, it is worth noting that, at least within our definition of natural SUSY, we can derive absolute lower bounds of $440 \mathrm{GeV}$ and $230 \mathrm{GeV}$ on the mass of the gluino and the lighter stop squark, respectively. These hold for all choices of the other parameters, in particular also for very compressed spectra. As noted above, monojet searches play an important role in deriving these absolute lower bounds.

\begin{tabular}{|l|l|}
\hline Type & Boundaries \\
\hline Allowed & $m_{\tilde{t}_{1}}>630 \mathrm{GeV}$ and $m_{\tilde{g}}>1150 \mathrm{GeV}$ \\
& $m_{\tilde{g}}>1100 \mathrm{GeV}$ and $m_{\tilde{\chi}_{1}^{0}}>320 \mathrm{GeV}$ \\
\hline Excluded & $m_{\tilde{t}_{1}}<260 \mathrm{GeV}$ or $m_{\tilde{g}}<480 \mathrm{GeV}$ \\
& $m_{\tilde{g}}<1040 \mathrm{GeV}$ and $m_{\tilde{\chi}_{1}^{0}}<340 \mathrm{GeV}$ \\
& $m_{\tilde{g}}<1070 \mathrm{GeV}$ and $m_{\tilde{\chi}_{1}^{0}}<200 \mathrm{GeV}$ \\
& $m_{\tilde{t}_{1}}<390 \mathrm{GeV}$ and $m_{\tilde{\chi}_{1}^{0}}<230 \mathrm{GeV}$ \\
\hline
\end{tabular}

TABLE V. List of allowed and excluded regions, where we now demand that in the allowed regions, all model points have $r<1$, while in excluded regions, all model points have $r>1$. There are no ambiguous points in this case.

Recall that we call a model point (clearly) excluded only if $r_{\max }>1.5$. This helped to separate excluded and allowed regions in the figures discussed in this Section. Nevertheless this may be overly conservative, since CheckMATE already incorporates the statistical Monte Carlo error of the simulation in the calculation of $r$. In Table $\mathrm{V}$ we therefore list "allowed" regions where all model points satisfy $r<1$ and "excluded" regions where all model points have $r>1$. Since there are no ambiguous points in this definition, the "not excluded" regions listed separately in Table IV] are then identical to the "allowed" regions, and are therefore not listed separately in Table V.

Comparing Table V with Table IV we see that both the allowed and the excluded regions have become larger, since the requirements defining these regions have become weaker. However, the allowed regions in Table $\mathrm{V}$ are still smaller than the corresponding "not excluded" regions in Table IV since the latter allow points with $r_{\max }$ up to 1.5 , while in the former all points have to satisfy $r<1$. In particular, the island of compressed spectra discussed in Fig. 8 and listed as third "not excluded" region in Table IV] does not appear in Table V. We saw in Fig. 8 that some points in this region have $r<2 / 3$; these points obviously also satisfy the requirement $r<1$ used to define an allowed model point in Table V. However, there are also model points throughout this island with $1<r<1.5$, which are now counted as excluded, making it impossible to define a contiguous allowed region defined in terms of only the gluino, lighter stop and LSP masses in this case. On the other hand, the other allowed and excluded regions in Table $\mathrm{V}$ do not differ too much from the corresponding regions in Table IV. In particular, 
the absolute lower bounds on the $\tilde{t}_{1}$ and gluino masses have increased by only about $10 \%$.

Finally, we want to investigate the impact of the decoupled sparticle spectrum on our results. Here, we want to have a closer look at the electroweak gaugino sector. As discussed in Section II. we have fixed the bino and wino mass parameters to rather large values, $M_{1}=M_{2}=3 \mathrm{TeV}$. As a result, the mass splitting between the second lightest neutralino or the chargino and the LSP (see Fig. 1) is too small to have an measurable effect on the LHC phenomenology. However, if the values of the gaugino mass parameters are significantly lowered, the mixing between the $U(1)$ as well as $S U(2)$ gauginos and the higgsinos will become larger and the mass gap between the second lightest neutralino or the lighter chargino mass eigenstate and the LSP will be widened. Hence, the decay products of the chargino and the second lightest neutralino might become energetic enough to be detected at the LHC.

We checked whether this is the case by varying the Bino mass parameter $M_{1}$ while keeping all other parameters the same. We then computed the optimal value of $r$ from gluino, stop and sbottom production independently, rather than summing over all three modes. This probes the $M_{1}$ dependence of three different signal regions. The results are depicted in Fig. 9 We used the same model point for the stop and sbottom pair production, with $m_{\tilde{t}_{1}} \simeq 380 \mathrm{GeV}, m_{\tilde{b}_{1}} \simeq 475$ $\mathrm{GeV}$ and $\mu=265 \mathrm{GeV}$. Recall that our input parameters are $\overline{\mathrm{DR}}$ parameters; the physical squark masses do therefore vary slightly when $M_{1}$ is changed, but the variation is well below $1 \%$. We used a different scenario to probe the $M_{1}$ dependence of the gluino pair production signal, with $\mu=135 \mathrm{GeV}, m_{\tilde{g}} \simeq 1085$ $\mathrm{GeV}, m_{\tilde{t}_{1}} \simeq 900 \mathrm{GeV}$ and heavy $\tilde{b}_{1}$ yielding $r \simeq 1.5$, since a possible $M_{1}$ dependence would be most important for us for scenarios with $r \simeq 1$, i.e. near the boundaries of the allowed and excluded regions. In all three cases we kept $M_{2}$ fixed to $3 \mathrm{TeV}$. It is clear from the plot that $r$ is constant within the error induced by the Monte Carlo statistics. We conclude that the exact values of $M_{1}$ and $M_{2}$ are not relevant for our results as long as $\left|M_{1}\right|,\left|M_{2}\right| \gg|\mu|$.

We conclude this Section with some comments on model points which are difficult to observe with run-1 SUSY searches. The simplest (and most obvious) reason for this is that the total production cross section before cuts becomes very small for heavy SUSY particles. Many of these model points will be tested by run-2 of the LHC, which is currently under way.

Some model points are kinematically accessible but still missed by all searches. We encountered one reason for this already: a relatively compressed spectrum, with small mass difference between the strongly interacting superparticles and the LSP, greatly reduces the cut efficiencies for most analyses. In the most extreme case one has to fall back on monojet searches, where the signal only occurs at $\mathcal{O}\left(\alpha_{S}^{3}\right)$ and suffers from a large irreducible background from $Z+$ jet production.

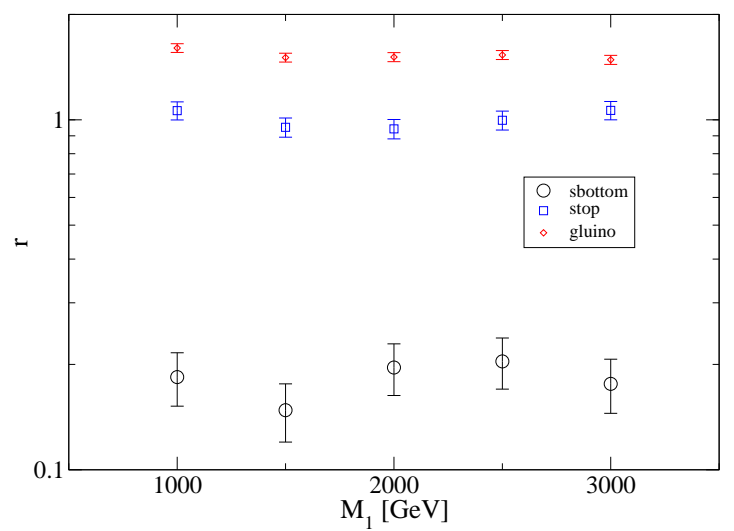

FIG. 9. The value of $r$ a function of the soft breaking parameter $M_{1}$ for the lighter stop, sbottom and gluino pair production. The other parameters are kept fixed. The results for stop and sbottom pair production have been computed with the same spectrum (but they probe different signal regions), while the results for gluino production are for a different model point, as explained in the text. The errors shown are due to Monte Carlo statistics.

Another class of difficult model points satisfies $m_{\tilde{t}_{1}} \sim m_{\tilde{\chi}_{1}^{0}}+m_{t}$, with $\tilde{t}_{1} \rightarrow t \tilde{\chi}_{1}^{0}$ being the dominant decay mode. In this case the LSPs in the final state often have small momenta, so that the event resembles a $t \bar{t}$ event. This leads to well-known holes in the ATLAS and CMS exclusion plots in the $\tilde{t}_{1}$ and LSP mass planes. This has been discussed in Sect. II], where we also pointed out that this scenario can only be approximated in our definition of natural supersymmetry: if the phase space for $\tilde{t}_{1} \rightarrow t \tilde{\chi}_{1}^{0}$ becomes too small, $\tilde{t}_{1} \rightarrow b \tilde{\chi}_{1}^{+}$decays will take over, which are always allowed if $\tilde{\chi}_{1}^{0}$ is higgsino-like with $m_{\tilde{\chi}_{1}^{0}} \simeq m_{\tilde{t}_{1}}-m_{t}$. One can try to suppress the branching ratio into the chargino mode by making $\tilde{t}_{1}$ mostly an $S U(2)$ doublet and chosing a small value of $\tan \beta$. However, the latter cannot be too small, since we insist on reproducing the observed mass of the Higgs boson. Moreover, a light doublet-like $\tilde{t}_{1}$ implies that $\tilde{b}_{1}$ is also relatively light, with mass typically 40 to $100 \mathrm{GeV}$ above $m_{\tilde{t}_{1}}$. Hence the top quarks from $\tilde{b}_{1}$ decay will have significant energy in the $\tilde{b}_{1}$ rest frame, so that $\tilde{b}_{1}$ pair production can be distinguished from top pair production. As a result, the smallest clearly allowed (ambiguous) $\tilde{t}_{1}$ mass for model points with $m_{\tilde{\chi}_{1}^{0}}<m_{\tilde{t}_{1}}-m_{t}-40$ $\mathrm{GeV}$ and branching ratio $B\left(\tilde{t}_{1} \rightarrow t \tilde{\chi}_{1,2}^{0}\right)>0.5$ is about $390(310) \mathrm{GeV}$ (with $m_{\tilde{b}_{1}}=470(430) \mathrm{GeV}$ ). Furthermore, all model points where $\tilde{t}_{1}$ is lighter than $450 \mathrm{GeV}$ and can decay into top plus neutralino have $r \geq 0.4$, and should thus be testable in future LHC runs. 


\section{SUMMARY AND CONCLUSIONS}

In this work, we have considered natural SUSY scenarios characterized by light higgsinos with $|\mu| \leq 500$ $\mathrm{GeV}$, relatively light third generation $S U(2)$ doublet squarks and singlet stops, $m_{\tilde{t}_{1(2)}}, m_{\tilde{b}_{1}} \leq 1.5 \mathrm{TeV}$, and gluinos with mass $m_{\tilde{g}} \leq 3 \mathrm{TeV}$ in order to preserve the electroweak hierarchy. The first and second generation squarks, all sleptons as well as the EW gauginos are decoupled; this avoids direct search limits from ATLAS and CMS and suppresses FCNC and CP violating processes. Since the observed Higgs couplings are consistent with the SM predictions, we work in the decoupling limit where all additional Higgs bosons predicted by the MSSM are also very heavy.

We have randomly generated 22000 natural SUSY model points in the six dimensional parameter space: $m_{\tilde{Q}_{t}}, m_{\tilde{t}_{R}}, \mu, M_{3}, A_{t}$ and $\tan \beta$ assuming flat priors. We demanded a SM like Higgs boson with $m_{h}=$ $125 \pm 3 \mathrm{GeV}$ and a neutralino LSP. In this setup, the higgsino mass eigenstates are always almost mass degenerate so that their decay products are too soft to be observed, making it essentially impossible to probe direct higgsino pair production at the LHC. On the other hand, stops, sbottoms as well as gluinos can be copiously produced at the LHC. Novel decay signatures such as heavier stop decays into $Z$ and $h$ or sbottom decays into $W$ bosons emerge but these decay modes cannot be probed with current LHC data since $m_{\tilde{t}_{2}}>800 \mathrm{GeV}$ is required in order to obtain a sufficiently heavy SM like Higgs and hence the heavier stop could not produced at an observable rate during LHC run-1.

We have generated signal events for each model point and have passed the event files to CheckMATE which provides a framework to test a model against a large number of current ATLAS and CMS $8 \mathrm{TeV}$ searches for beyond the SM physics, in particular, SUSY searches. We included the results of searches optimized for simplified natural SUSY scenarios, of direct squark and gluino searches and of inclusive SUSY searches in our scan. All these searches have been implemented and fully validated in CheckMATE. We have found that nearly all the searches we include indeed exclude some model points, and eight different searches provide the best sensitivity in some region of parameter space. This shows that considering a large number of different searches is indeed necessary in order to determine whether a model point is allowed or not.

The main results of our analysis are summarized in Tables IV] and V, which delineate allowed and excluded regions in parameter space. In particular, we found that all scenarios where either $m_{\tilde{t}_{1}}<230 \mathrm{GeV}$ or $m_{\tilde{g}}<440 \mathrm{GeV}$ are clearly excluded, irrespective of the values of the other parameters. On the other hand, all model points with $m_{\tilde{t}_{1}}>660 \mathrm{GeV}$ and $m_{\tilde{g}}>1180$ $\mathrm{GeV}$ are currently still clearly allowed. Here we call a model point "clearly allowed" only if its predicted signal in the signal region which is expected to be most sensitive to this point is at least 1.5 times the nominal 95\% c.l. upper bound, while "clearly allowed" points have the a predicted signal in this "optimal" signal region which is at least a factor 1.5 below the nominal bound. This serves to avoid overlap of allowed and excluded regions due to Monte Carlo fluctuations. Note also that we did not include any theoretical uncertainty of our prediction beyond the statistical error of our Monte Carlo simulation. This factor of 1.5 can thus also be interpreted as a (rather conservative) estimate of the additional theory uncertainty. In many cases it is sufficient to specify the masses of the lighter stop, the gluino, and the LSP in order to decide whether a parameter point is excluded or allowed, but in some cases the values of the other parameters are also important. In particular, there are significant differences between points with doublet-like or singletlike lighter stop, not least because a doublet-like light stop also implies a rather light sbottom. Moreover, we found some cases where small differences in parameters can lead to large differences in the ratio of the expected signal in the most sensitive search region to its upper bound.

Overall we find that a large part of the parameter space of our definition of natural supersymmetry is still allowed. Much of this parameter space can be explored by run-2 of the LHC, which just started. We forecast that inclusive SUSY searches will play an increasingly prominent role in exploring the remaining parameter space, while dedicated searches for final states containing top or bottom quarks will play a lesser role than in the analysis of run-1 data. However, most likely again many analyses, and an even larger number of signal regions, will have to be combined in order to comprehensively probe the remaining parameter space. We look forward to the results of the ongoing run.

\section{ACKNOWLEDGMENTS}

We thank Sabine Kraml for discussions. The work by M.D. was partially supported by the BMBF Theorieverbund. The work of J.S.K. was partially supported by the MINECO, Spain, under contract FPA2013-44773-P; Consolider-Ingenio CPAN CSD2007-00042 and the Spanish MINECO Centro de excelencia Severo Ochoa Program under grant SEV2012-0249. J.S.K. would like to thank Bonn university for support and hospitality while part of this manuscript was prepared.

\section{Appendix A: Analyses}

Here we give brief descriptions of the analyses we used in our scan. We focus on those aspects that are relevant for our definition of natural SUSY, although our model points are also tested against signal regions 
that are optimized for decay chains that cannot be realized in our set-up.

\section{1. $\quad 1308.2631$ (ATLAS)}

This analysis 51 concentrates on signatures with two $b$-jets and missing transverse momentum. It was optimized for sbottom pair production followed by $\tilde{b}_{1} \rightarrow b \tilde{\chi}_{1}^{0}$. A similar final state arises from stop pair production followed by $\tilde{t}_{1} \rightarrow \tilde{\chi}_{1}^{+} b$. The search has two signal regions targeting scenarios with a large mass splitting between the squark and the LSP or with a compressed spectrum. The former signal region demands large transverse momentum and two $b$-jets and the latter requires a leading non $b$ jet recoiling against the squark pair system with two $b$ tagged jets and large transverse momentum.

\section{2. $\quad 1403.4853$ (ATLAS)}

This search for direct stop pair production in final states with two leptons and large missing transverse momentum 52 targets scenarios with $\tilde{t} \rightarrow \tilde{\chi}_{1}^{ \pm} b$ with $\Delta m\left(\tilde{\chi}_{1}^{ \pm}, \tilde{\chi}_{1}^{0}\right) \geq m_{W}$, or $\tilde{t} \rightarrow t \tilde{\chi}_{1}^{0}$ with an on (off)-shell top quark. $t \bar{t}$ and $W^{+} W^{-}$production are the main background processes to this search and the stransverse mass $m_{T 2}$ observable [53] can be used to suppress these backgrounds very efficiently. The signal regions targeting $\tilde{t}_{1} \rightarrow b \chi_{1}^{ \pm}$are divided according to jet multiplicity and $m_{T 2}$, whereas one signal region explicitly requires $2 b$-jets. The on-shell $\tilde{t}_{1} \rightarrow t \tilde{\chi}_{1}^{0}$ mode is addressed via a multivariate method and is not implemented in CheckMATE.

\section{3. $\quad 1404.2500$ (ATLAS)}

This analysis 54 considers final states containing two same sign leptons or at least three leptons. This search was optimized for gluino mediated stop production, $\tilde{g} \rightarrow t_{1} \tilde{t}$ with $\tilde{t}_{1} \rightarrow t \tilde{\chi}_{1}^{0}$. Here, one can expect up to four leptons in the final state. The selection requirements of the five signal regions differ in the number of $b$-tagged jets, jet multiplicity, missing transverse momentum cut, threshold of the effective mass, and the transverse mass computed from the $p_{T}$ of the hardest lepton and the missing $p_{T}$.

\section{1407.0583 (ATLAS)}

This analysis [55] is designed to search for final states containing one lepton, a minimum of two jets and large transverse missing momentum. The study contains fifteen signal regions targeting a large number of stop pair production scenarios, with subsequent decay modes such as $\tilde{t}_{1} \rightarrow t \tilde{\chi}_{1}^{0}, \tilde{t}_{1} \rightarrow b \tilde{\chi}_{1}^{ \pm}, \tilde{t}_{1} \rightarrow b f f^{\prime} \tilde{\chi}_{1}^{0}$, $\tilde{t}_{1} \rightarrow b W \tilde{\chi}_{1}^{0}$, and non-symmetric decay modes such as $\tilde{t}_{1} \rightarrow t \tilde{\chi}_{1}^{0}, \tilde{t}_{1}^{*} \rightarrow \bar{b} \tilde{\chi}_{1}^{-}$. All signal regions include a veto on a second lepton. The signal regions optimized for $\tilde{t}_{1} \rightarrow t \tilde{\chi}_{1}^{0}$ decays use shape information of the large missing transverse momentum and transverse mass distribution. If the mass difference between the stop and neutralino is very large, the top quark can be boosted and large-cone jets are used. The signal regions targeting $\tilde{t}_{1} \rightarrow b \tilde{\chi}_{1}^{ \pm}$decays require different kinematic cuts on the leptons, $(b-)$ jets, missing transverse momentum, transverse mass, asymmetric stransverse mass and $b$-jet multiplicity or vetoes on isolated tracks and hadronic taus.

\section{5. $\quad 1407.0608$ (ATLAS)}

In scenarios where $\tilde{t}_{1}$ has small mass splitting to the LSP the stop decay mode $\tilde{t}_{1} \rightarrow b \tilde{\chi}_{1}^{0} W$ can be kinematically closed, while the four-body decay $\tilde{t}_{1} \rightarrow \ell \nu_{\ell} b \tilde{\chi}_{1}^{0}$ is strongly suppressed because it is a third order process which is very sensitive to phase space. Thus the loopinduced decay $\tilde{t}_{1} \rightarrow c \tilde{\chi}_{1}^{0}$ can be the dominant decay mode [30. This analysis [56] is optimized for searches for stop pair production with $\tilde{t}_{1} \rightarrow c \tilde{\chi}_{1}^{0}$. The study has defined two classes of signal regions. Both sets have the same preselection cuts which require a hard jet, large missing transverse momentum and a lepton veto. The first class of signal regions targets scenarios with a very small mass splitting between the stop and the neutralino LSP and thus the charm jets are too soft to be reconstructible. The selection cuts thus isolate monojet events. The second set of signal regions considers non-degenerate scenarios and exploits a dedicated charm tagging algorithm. The signal regions are further divided by the applied cuts on the momentum of the leading jet and on the missing transverse momentum.

\section{6. $\quad 1303.2985$ (CMS)}

This analysis is designed to be sensitive to hadronic final states with missing transverse energy using the variable $\alpha_{T}$ [57]. The sensitivity of the search is improved by categorizing events according to the multiplicities of $b$-tagged and other jets. The signal regions span a wide range of cuts on the scalar sum of transverse energies of all the jets. Hence, the search is sensitive to a large number of third generation simplified models such as gluino mediated stop and sbottom production and direct stop and/or sbottom production followed by their hadronic decay.

\section{ATLAS-CONF-2012-104}

This search targets final states with at least four hard jets, missing transverse momentum and one lep- 
ton, and uses an integrated luminosity of only $5.8 \mathrm{fb}^{-1}$ [58. The study has two non-overlapping search regions corresponding to an electron and a muon channel. The event selection is mainly based on the transverse mass of the lepton and missing transverse momentum, as well as on the inclusive mass defined as the scalar sum of the transverse momenta of the lepton, the jets and the missing transverse momentum. The cuts are designed to efficiently suppress the dominant $t \bar{t}$ and $W / Z+$ jet backgrounds. The search results were interpreted as limits on the parameter space of minimal supergravity scenarios. In particular, the search is motivated by scenarios where a left handed squark dominantly decays to a light chargino. However, the search is also sensitive to natural SUSY scenarios, such as gluino production with subsequent decays into $t \bar{b} \tilde{\chi}_{1}^{-}$ or into stop and top with semileptonic decay of the top and hadronic stop decay.

\section{ATLAS-CONF-2013-024}

This analysis searches for direct production of the top squark with subsequent decay into top plus LSP [59. It concentrates on the purely hadronic decay mode of the top quark and thus the all-hadronic stop search demands six or more jets while at least two jets are tagged as $b$-jets. Each event is required to be consistent with containing two top quarks and thus two 3-jet systems must each have an invariant mass consistent with the top quark mass. The signal definition includes a lepton as well as a tau veto and a considerable amount of transverse missing momentum is required. The search is divided into three signal regions with increasing minimum missing transverse momentum cuts.

\section{ATLAS-CONF-2013-047}

This analysis 60] is designed to look for heavy squark and gluino production in final states with high momentum jets, large missing transverse momentum and no leptons, using a total integrated luminosity of $20.3 \mathrm{fb}^{-1}$. The null results are interpreted as limits on simplified models in the gluino (squark) and neutralino LSP mass plane as well as in mSUGRA/CMSSM parameter space. Since the search aims for heavy squark and gluino production modes, $m_{\text {eff }}$ is a powerful observable to separate the signal from the SM background. $m_{\mathrm{eff}}$ is defined as the scalar sum of the transverse momenta of the jets in the final state plus the missing transverse momentum. The large number of signal regions with differing jet multiplicity and kinematic requirements allow to target a broad range of squark and gluino models from short to long cascade decays. As no $b$-jet veto is applied, the search is also sensitive to natural SUSY models as long as high $p_{T}$ jets and large missing transverse momentum are expected in the final state.

\section{ATLAS-CONF-2013-061}

This multi $b$-jets study 61 aims at final states with four or more jets, at least three of which originate from $b$-quarks, and large missing transverse momentum. It uses an integrated luminosity of $20.1 \mathrm{fb}^{-1}$. The signal regions are defined via the number of charged leptons (zero or $\geq 1$ ), the jet multiplicity, as well as different kinematic requirements on jet momentum, missing transverse momentum and the effective mass. In 61] the search results are interpreted in the context of simplified natural SUSY scenarios and in the context of mSUGRA/CMSSM scenarios, but this search is also very powerful in constraining natural SUSY models since gluino pair production there frequently leads to final states with four $b$-quarks. Several signal regions target gluino decays into $\tilde{g} \rightarrow \tilde{b} b$ or $\tilde{g} \rightarrow \tilde{t} t$, while others consider scenarios with gluinos decaying via off-shell third generation squarks.

\section{ATLAS-CONF-2013-062}

This analysis 62 focuses on searches for squarks and gluinos in final states with isolated (soft) leptons, jets and missing transverse momentum, using a data set corresponding to an integrated luminosity of $20 \mathrm{fb}^{-1}$. Limits are derived on simplified gluino and stop pair production scenarios as well on the mSUGRA/CMSSM model. This search is divided into five classes of signal regions which are based on the inclusive hard single lepton channel, a soft single lepton channel optimized for compressed spectra, the soft dimuon channel addressing the mUED model and soft single lepton signal regions with one or two $b$-jets targeting stop pair production for small and moderate mass splitting between the stop and the neutralino LSP, respectively.

\section{CMS-SUS-13-016 (CMS)}

This analysis 63] searches for superparticle production in events with two opposite sign leptons, a large number of jets, $b$-tagged jets, and large missing transverse energy. This search is designed to search for gluino pair production with $\tilde{g} \rightarrow t \bar{t} \tilde{\chi}_{1}^{0}$. It only has one signal region which demands at least five jets, three of which are $b$-tagged, and large missing transverse momentum. 
[1] M. Drees, R.M. Godbole and P. Roy, "Theory and Phenomenology of Sparticles", World Scientific, Singapore (2004); H. Baer and X.R. Tata, "Weak scale supersymmetry: From superfields to scattering events", Cambridge University Press (2006).

[2] L. E. Ibáñez and G. G. Ross, Nucl. Phys. B 368 (1992) 3.

[3] J. S. Kim, K. Rolbiecki, K. Sakurai and J. Tattersall, JHEP 1412 (2014) 010 arXiv:1406.0858 [hep-ph]].

[4] B. Allanach, S. Biswas, S. Mondal and M. Mitra, Phys. Rev. D 91 (2015) 1, 011702 arXiv:1408.5439 [hep-ph]].

[5] A. H. Chamseddine, R. L. Arnowitt and P. Nath, Phys. Rev. Lett. 49 (1982) 970.

[6] J. Alwall, M. P. Le, M. Lisanti and J. G. Wacker, Phys. Rev. D 79 (2009) 015005 arXiv:0809.3264 [hep-ph]].

[7] G. Aad et al. [ATLAS Collab.], arXiv:1507.05525 [hepex].

[8] G. Aad et al. [ATLAS and CMS Collab.s], Phys. Rev. Lett. 114 (2015) 191803 arXiv:1503.07589 [hep-ex]].

[9] J.R. Ellis, K. Enqvist, D. V. Nanopoulos and F. Zwirner. Mod. Phys. Lett. A1 (1986) 57; R. Barbieri and G. F. Giudice, Nucl. Phys. B 306 (1988) 63.

[10] J. L. Feng, K. T. Matchev and T. Moroi, Phys. Rev. Lett. 84 (2000) 2322 hep-ph/9908309.

[11] R. Kitano and Y. Nomura, Phys. Rev. D 73 (2006) 095004 hep-ph/0602096.

[12] M. Papucci, J.T. Ruderman and A. Weiler, JHEP 1209 (2012) 035 arXiv:1110.6926 [hep-ph]].

[13] J. A. Casas, J. M. Moreno, S. Robles, K. Rolbiecki and B. Zaldvar, JHEP 1506 (2015) 070 arXiv:1407.6966 [hep-ph]].

[14] H. Baer, V. Barger, P. Huang, A. Mustafayev and X. Tata, Phys. Rev. Lett. 109 (2012) 161802 arXiv:1207.3343 [hep-ph]], and Phys. Rev. D87 (2013) 11, 115028 arXiv:1212.2655 [hep-ph]].

[15] G. Aad et al. [ATLAS Collab.], arXiv:1506.08616 [hepex].

[16] S. Chatrchyan et al. [CMS Collab.], Eur. Phys. J. C 73 (2013) 12, 2677 arXiv:1308.1586 [hep-ex]].

[17] H. Baer, V. Barger, P. Huang and X. Tata, JHEP 1205 (2012) 109 arXiv:1203.5539 [hep-ph]].

[18] O. Buchmueller and J. Marrouche, Int. J. Mod. Phys. A 29 (2014) 06, 1450032 arXiv:1304.2185 [hep-ph]].

[19] C. Han, K.-i. Hikasa, L. Wu, J. M. Yang and Y. Zhang, JHEP 1310 (2013) 216 arXiv:1308.5307 [hep-ph]].

[20] G. Bélanger, D. Ghosh, R. Godbole and S. Kulkarni, arXiv:1506.00665 [hep-ph].

[21] A. Kobakhidze, N. Liu, L. Wu, J. M. Yang and M. Zhang, arXiv:1511.02371 [hep-ph].

[22] K. Kowalska and E. M. Sessolo, Phys. Rev. D 88 (2013) 7, 075001 doi:10.1103/PhysRevD.88.075001 arXiv:1307.5790 [hep-ph]].

[23] G. Gamberini, G. Ridolfi and F. Zwirner, Nucl. Phys. B 331 (1990) 331.

[24] F. Gabbiani, E. Gabrielli, A. Masiero and L. Silvestrini, Nucl. Phys. B $\mathbf{4 7 7}$ (1996) 321 hep$\mathrm{ph} / 9604387$.

[25] D.S.M. Alves, M.R. Buckley, P.J. Fox, J.D. Lykken and C.-T. Yu, Phys. Rev. D87 (2013) 035016 arXiv:1205.5805 [hep-ph]]; M.R. Buckley, T. Plehn and M.J. Ramsey-Musolf, Phys. Rev. D90 (2014)
014046 arXiv:1403.2726 [hep-ph]].

[26] J.R. Ellis, T. Falk and K.A. Olive, Phys. Lett. B444 (1998) 367 hep-ph/9810360.

[27] K.J. Bae, H. Baer and E.J. Chun, Phys. Rev. D89 (2014) 3, 031701 arXiv:1309.0519 [hep-ph]].

[28] C.H. Chen, M. Drees and J.F. Gunion, Phys. Rev. D55 (1997) 330; erratum, Phys. Rev. D60 (1999) 039901 hep-ph/9607421.

[29] H. Baer, A. Mustafayev and X. Tata, Phys. Rev. D89 (2014) 055007 arXiv:1401.1162 [hep-ph]].

[30] K.-i. Hikasa and M. Kobayashi, Phys. Rev. D 36 (1987) 724.

[31] H. Baer, X. Tata and J. Woodside, Phys. Rev. D 42 (1990) 1568.

[32] G. Chalons and D. Sengupta, arXiv:1508.06735 [hep$\mathrm{ph}$.

[33] W. Porod and F. Staub, Comput. Phys. Commun. 183 (2012) 2458 arXiv:1104.1573 [hep-ph]].

[34] J. Alwall, M. Herquet, F. Maltoni, O. Mattelaer and T. Stelzer, JHEP 1106 (2011) 128 arXiv:1106.0522 [hep-ph]].

[35] T. Sjöstrand, S. Mrenna and P. Z. Skands, JHEP 0605 (2006) 026 hep-ph/0603175.

[36] T. Sjüstrand et al., Comput. Phys. Commun. 191 (2015) 159 arXiv:1410.3012 [hep-ph]].

[37] M. Drees, H. Dreiner, D. Schmeier, J. Tattersall and J. S. Kim, Comput. Phys. Commun. 187 (2014) 227 arXiv:1312.2591 [hep-ph]].

[38] https://checkmate.hepforge.org/

[39] J. S. Kim, D. Schmeier, J. Tattersall and K. Rolbiecki, Comput. Phys. Commun. 196 (2015) 535 arXiv:1503.01123 [hep-ph]].

[40] J. de Favereau et al. [DELPHES 3 Collab.], JHEP 1402 (2014) 057 arXiv:1307.6346 [hep-ex]].

[41] S. Chatrchyan et al. [CMS Collab.], JHEP 1307 (2013) 122 arXiv:1305.0491 [hep-ex]]; G. Aad et al. [ATLAS Collab.], Eur. Phys. J. C75 (2015) 407 arXiv:1506.05332 [hep-ex]];

[42] M. Drees and K. Hagiwara, Phys. Rev. D 42 (1990) 1709.

[43] G. Abbiendi et al. [OPAL Collab.], Eur. Phys. J. C 29 (2003) 479 hep-ex/0210043.

[44] J. Abdallah et al. [DELPHI Collab.], Eur. Phys. J. C 31 (2003) 421 hep-ex/0311019.

[45] G. Abbiendi et al. [OPAL Collab.], Eur. Phys. J. C 35 (2004) 1 hep-ex/0401026.

[46] M. Carena, A. Freitas and C. E. M. Wagner, JHEP 0810 (2008) 109 arXiv:0808.2298 [hep-ph]]; M. Drees, M. Hanussek and J.S. Kim, Phys. Rev. D86 (2012) 035024 arXiv:1201.5714 [hep-ph]].

[47] M. L. Mangano, M. Moretti, F. Piccinini and M. Treccani, JHEP 0701 (2007) 013 hep-ph/0611129.

[48] Measuring the b-tag efficiency in a top-pair sample with $4.7 \mathrm{fb}-1$ of data from the ATLAS detector, Tech. Rep. ATLAS-CONF-2012-097, CERN, Geneva, Jul 2012.

[49] Measurement of the b-tag Efficiency in a Sample of Jets Containing Muons with $5 \mathrm{fb} 1$ of Data from the ATLAS Detector, Tech. Rep. ATLAS-CONF-2012043, CERN, Geneva, Mar 2012.

[50] A. L. Read, J. Phys. G 28 (2002) 2693.

[51] G. Aad et al. [ATLAS Collab.], JHEP 1310 (2013) 
189 arXiv:1308.2631 [hep-ex]].

[52] G. Aad et al. [ATLAS Collab.], JHEP 1406 (2014) 124 [arXiv:1403.4853 [hep-ex]].

[53] C. G. Lester and D. J. Summers, Phys. Lett. B 463 (1999) 99 hep-ph/9906349.

[54] G. Aad et al. [ATLAS Collab.], JHEP 1406 (2014) 035 arXiv:1404.2500 [hep-ex]].

[55] G. Aad et al. [ATLAS Collab.], JHEP 1411 (2014) 118 arXiv:1407.0583 [hep-ex]].

[56] G. Aad et al. [ATLAS Collab.], Phys. Rev. D 90 (2014) 5, 052008 arXiv:1407.0608 [hep-ex]].

[57] S. Chatrchyan et al. [CMS Collab.], Eur. Phys. J. C 73 (2013) 9, 2568 arXiv:1303.2985 [hep-ex]].

[58] G. Aad et al. [ATLAS Collab.], Tech. Rep. ATLAS-
CONF-2012-104, CERN, Geneva, Aug 2012.

[59] G. Aad et al. [ATLAS Collab.], Tech. Rep. ATLASCONF-2013-024, CERN, Geneva, Mar 2013.

[60] G. Aad et al. [ATLAS Collab.], Tech. Rep. ATLASCONF-2013-047, CERN, Geneva, May 2013.

[61] G. Aad et al. [ATLAS Collab.], Tech. Rep. ATLASCONF-2013-061, CERN, Geneva, Jun 2013.

[62] G. Aad et al. [ATLAS Collab.], Tech. Rep. ATLASCONF-2013-062, CERN, Geneva, Jun 2013.

[63] S. Chatrchyan et al. [CMS Collab.], CMS-PAS-SUS13016, CERN, Geneva, November, 2013.

[64] See e.g. the chapter on Statistics in K.A. Olive et al. (Particle Data Group), Chin. Phys. C38 (2014) 090001. 